\title{
HOMOGENEOUS VECTOR BUNDLES OVER ABELIAN VARIETIES VIA REPRESENTATION THEORY
}

\author{
MICHEL BRION
}

\begin{abstract}
Let $A$ be an abelian variety over a field. The homogeneous (or translation-invariant) vector bundles over $A$ form an abelian category $\mathrm{HVec}_{A}$; the Fourier-Mukai transform yields an equivalence of $\mathrm{HVec}_{A}$ with the category of coherent sheaves with finite support on the dual abelian variety. In this paper, we develop an alternative approach to homogeneous vector bundles, based on the equivalence of $\mathrm{HVec}_{A}$ with the category of finite-dimensional representations of a commutative affine group scheme (the "affine fundamental group" of $A$ ). This displays remarkable analogies between homogeneous vector bundles over abelian varieties and representations of split reductive algebraic groups.
\end{abstract}

\section{Contents}

1. Introduction

Notation and conventions

2. Universal affine covers and homogeneous bundles

2.1. Commutative quasi-compact group schemes

2.2. Universal affine covers of abelian varieties

2.3. Applications to homogeneous vector bundles

3. Representations of commutative affine group schemes

3.1. Irreducible representations

3.2. Blocks

4. Isogenies

4.1. Functorial properties of universal affine covers 106

4.2. Unipotent vector bundles $\quad 110$

\begin{tabular}{ll}
\hline 112 \\
\hline 113
\end{tabular}

References 113

\section{INTRODUCTION}

Consider an abelian variety $A$ over a field $k$. A vector bundle over $A$ is said to be homogeneous if it is invariant under pullback by translations in $A$; for instance, the homogeneous line bundles are parameterized by $\operatorname{Pic}^{0}(A)=\widehat{A}(k)$, where $\widehat{A}$ denotes the dual abelian variety. The homogeneous vector bundles have been extensively studied when $k$ is algebraically closed. As shown by Mukai, they form an abelian category $\mathrm{HVec}_{A}$ which is equivalent to the category of coherent sheaves with finite

Received by the editors June 12, 2018, and, in revised form, December 5, 2019.

2010 Mathematics Subject Classification. Primary 14J60, 14K05; Secondary 14L15, 20 G05. 
support on $\widehat{A}$ via the Fourier-Mukai transform. Moreover, any homogeneous vector bundle has a canonical decomposition

$$
E=\bigoplus_{L \in \widehat{A}(k)} L \otimes U_{L},
$$

where each $U_{L}$ is a unipotent vector bundle, i.e., an iterated extension of trivial line bundles; the unipotent bundles form an abelian subcategory $\mathrm{UVec}_{A}$, equivalent to that of coherent sheaves on $\widehat{A}$ with support at the origin (see [Mu78, §4] for these results). Also, the homogeneous vector bundles can be characterized as the iterated extensions of algebraically trivial line bundles (a result of Miyanishi; see [Mi73, §2]), or as the numerically flat vector bundles (this is due to Langer; see [La12, §6]).

In this paper, we develop an alternative approach to homogeneous vector bundles via representation theory, over an arbitrary ground field $k$. The starting point is again a result of [Mi73]: every such bundle is an associated vector bundle $G \times{ }^{H} V \rightarrow$ $G / H=A$ for some extension of algebraic groups $1 \rightarrow H \rightarrow G \rightarrow A \rightarrow 1$ with $H$ affine and some finite-dimensional $H$-module $V$. To handle all homogeneous vector bundles simultaneously, we take the inverse limit of all such extensions; this yields the universal extension of $A$ by an affine $k$-group scheme,

$$
1 \longrightarrow H_{A} \longrightarrow G_{A} \longrightarrow A \longrightarrow 1,
$$

where $G_{A}$ is a quasi-compact $k$-group scheme (see [EGA, IV .8.2.3]). We show that the associated vector bundle construction $V \mapsto G_{A} \times{ }^{H_{A}} V$ yields an equivalence of categories

$$
\mathcal{L}_{A}: H_{A} \text {-mod } \stackrel{\simeq}{\longrightarrow} \mathrm{HVec}_{A},
$$

where $H_{A}$-mod denotes the category of finite-dimensional $H_{A}$-modules (Theorem 2.9). Moreover, $\mathcal{L}_{A}$ induces isomorphisms of extension groups

$$
\operatorname{Ext}_{H_{A}}^{i}(V, W) \stackrel{\simeq}{\longrightarrow} \operatorname{Ext}_{A}^{i}\left(\mathcal{L}_{A}(V), \mathcal{L}_{A}(W)\right)
$$

for all $i \geq 0$ and all finite-dimensional $H_{A}$-modules $V, W$ (Theorem 2.12). In particular, homogeneity is preserved under extensions of vector bundles.

The $k$-group schemes $G_{A}$ and $H_{A}$ are not of finite type, but their structure is rather well-understood (see $[\operatorname{Br} 18, \S 3.3]$ and $\$ 2.2$ ). In particular, $G_{A}$ is commutative and geometrically integral; its formation commutes with base change under arbitrary field extensions. Moreover, $H_{A}$ is an extension of a unipotent group scheme $U_{A}$ by the group scheme of multiplicative type $M_{A}$ with Cartier dual $\widehat{A}(\bar{k})$. Also, $U_{A}$ is a vector group of dimension $g=\operatorname{dim}(A)$ if $k$ has characteristic 0 ; in positive characteristics, $U_{A}$ is profinite (see $\$ 2.2$ again). By Theorem 4.8, the equivalence of categories (1.3) induces an equivalence

$$
U_{A} \text { mod } \stackrel{\simeq}{\longrightarrow} \mathrm{UVec}_{A} .
$$

Homogeneous vector bundles are also preserved under tensor product and duality; clearly, these operations on $\mathrm{HVec}_{A}$ correspond via (1.3) to the tensor product and duality of $H_{A}$-modules (the description of these operations in terms of FourierMukai transform is more involved; see [Mu78, Thm. 4.12]). Also, homogeneity is preserved by pushforward and pullback under isogenies; we show that these correspond again to natural operations on modules, in view of a remarkable invariance property of the extension (1.2). More specifically, let $\varphi: A \rightarrow B$ be an isogeny of 
abelian varieties, with kernel $N$. Then $\varphi$ induces an isomorphism $G_{A} \stackrel{\simeq}{\longrightarrow} G_{B}$ and an exact sequence

$$
0 \longrightarrow H_{A} \longrightarrow H_{B} \longrightarrow N \longrightarrow 0
$$

(Proposition 4.1). Moreover, the pushforward (resp., pullback) under $\varphi$ yields an exact functor $\varphi_{*}: \mathrm{HVec}_{A} \rightarrow \mathrm{HVec}_{B}$ (resp., $\varphi^{*}: \mathrm{HVec}_{B} \rightarrow \mathrm{HVec}_{A}$ ), which may be identified with the induction, $\operatorname{ind}_{H_{A}}^{H_{B}}$ (resp., the restriction, $\operatorname{res}_{H_{A}}^{H_{B}}$ ) via the equivalences of categories $\mathcal{L}_{A}, \mathcal{L}_{B}$ (Theorem 4.2).

In particular, the homogeneous vector bundle $\varphi_{*}\left(\mathcal{O}_{A}\right)$ on $B$ corresponds to the $H_{B}$-module $\mathcal{O}(N)$, where $H_{B}$ acts via the regular representation of its quotient group $N$; thus, the indecomposable summands of $\varphi_{*}\left(\mathcal{O}_{A}\right)$ correspond to the blocks of the finite group scheme $N$. When applied to the $n$th relative Frobenius morphism in characteristic $p$, this yields a refinement of a recent result of Sannai and Tanaka ([ST16, Thm. 1.2]; see Remark 4.11 for details).

More generally, the category $H_{A}$-mod decomposes into blocks associated with irreducible representations of $H_{A}$, since every extension of two non-isomorphic such representations is trivial (see 4.1 ). This translates into a decomposition of the category $\mathrm{HVec}_{A}$ which generalizes (1.1); the line bundles $L \in \widehat{A}(k)$ are replaced with irreducible homogeneous vector bundles, parameterized by the orbits of the absolute Galois group in $\widehat{A}(\bar{k})$ (see $\$ 4.1$ again). We obtain a description of the block $\operatorname{HVec}_{A, x}$ associated with a point $x \in \widehat{A}(\bar{k})$, which takes a simpler form when the residue field $K:=k(x)$ is separable over $k$; then $\operatorname{HVec}_{A, x} \simeq \operatorname{UVec}_{A_{K}}=\operatorname{HVec}_{A_{K}, 0}$ (Theorem 4.8).

In addition to these results, the paper contains developments in two independent directions. First, we consider the universal affine covers of abelian varieties in the setting of quasi-compact group schemes (\$2.1). These covers are interesting objects in their own right; over an algebraically closed field, they were introduced and studied by Serre as projective covers in the abelian category of commutative proalgebraic groups (see [Se60, §9.2], and [Br18, §3.3] for a generalization to an arbitrary ground field). They also occur in very recent work of Ayoub on motives (see Ay19, §5.9]); moreover, the corresponding affine fundamental group, i.e., the group $H_{A}$ in the exact sequence (1.2), coincides with the $S$-fundamental group of $A$ as defined in La11 (see La12, $\S 6]$ ). We show that the group $G_{A}$ is the projective cover of $A$ in the abelian category of commutative quasi-compact group schemes; as a consequence, that category has enough projectives (Proposition 2.3). This builds on results of Perrin about (not necessarily commutative) quasi-compact group schemes, see Pe75, Pe76, and this follows a suggestion of Grothendieck in [EGA, IV.8.13.6] 1

The second development, in $₫ 3$ (which can be read independently of the rest of the paper), investigates the representation theory of a commutative affine group scheme $H$ over a field $k$. This is easy and well known when $k$ is algebraically closed (see, e.g., [Ja03, I.3.11]); the case of a perfect field $k$ follows readily by using Galois descent and the splitting $H=U \times M$, where $U$ is unipotent and $M$ of multiplicative type. But the affine fundamental group $H_{A}$ over an imperfect field admits no such

\footnotetext{
1 "Les seuls pro-groupes algébriques rencontrés en pratique jusqu'à présent étant en fait essentiellement affines, il y aura sans aucun doute avantage à substituer à l'étude des groupes pro-algébriques généraux (introduits et étudiés par Serre [Se60]) celle des schémas en groupes quasi-compacts sur $k$, dont la définition est conceptuellement plus simple."
} 
splitting (Lemma 2.6); this motivates our study of a topic which seems to have been unexplored.

This representation-theoretic approach displays remarkable analogies between homogeneous vector bundles over an abelian variety $A$ and over a full flag variety $X=G / B$, where $G$ is a split reductive algebraic group and $B$ a Borel subgroup: $G_{A}, H_{A}$ play similar roles as $G, B$; the group $\widehat{A}(\bar{k})$ is replaced with the weight lattice, and the Galois group with the Weyl group, etc. But these analogies are incomplete, as the combinatorics associated with root data of reductive groups have no clear counterpart on the side of abelian varieties; also, the block decompositions behave very differently.

Notation and conventions. Throughout this paper, we fix a ground field $k$ of characteristic $p \geq 0$. We choose an algebraic closure $\bar{k}$ of $k$, and denote by $k_{s}$ the separable closure of $k$ in $\bar{k}$. The Galois group of $k_{s} / k$ is denoted by $\Gamma$.

We consider schemes over $k$ unless otherwise mentioned; morphisms and products of schemes are understood to be over $k$ as well. Given a scheme $S$ and a field extension $K / k$, we denote by $S_{K}$ the scheme obtained from $S$ by the corresponding base change. We freely identify line bundles (resp., vector bundles) over a scheme with invertible sheaves (resp., locally free sheaves).

A variety is a separated, geometrically integral scheme of finite type. An algebraic group is a group scheme of finite type.

We denote by $A$ a non-zero abelian variety, and set $g:=\operatorname{dim}(A)$; as usual, the group law of $A$ is denoted additively, with neutral element $0 \in A(k)$. We use the notation $n_{A}$ for the multiplication by an integer $n$ in $A$, and $A[n]$ for its (schemetheoretic) kernel. The dual abelian variety is denoted by $\widehat{A}$.

\section{UNIVERSAL AFFINE COVERS AND HOMOGENEOUS BUNDLES}

2.1. Commutative quasi-compact group schemes. Let $\mathcal{C}$ be the category with objects the commutative algebraic groups, and morphisms the homomorphisms of group schemes; then $\mathcal{C}$ is an abelian category (see [SGA3, VIA.5.4.2]). Denote by $\mathcal{L}$ the full subcategory of $\mathcal{C}$ with objects the affine (or equivalently, linear) algebraic groups; then $\mathcal{L}$ is an abelian subcategory of $\mathcal{C}$, stable under taking subobjects, quotients, and extensions. We say that $\mathcal{L}$ is a Serre subcategory of $\mathcal{C}$. The full subcategory $\mathcal{F}$ of $\mathcal{C}$ with objects being the finite group schemes is a Serre subcategory of $\mathcal{L}$.

Next, consider the category $\widetilde{\mathcal{C}}$ with objects the commutative quasi-compact group schemes; the morphisms in $\widetilde{\mathcal{C}}$ are still the homomorphisms of group schemes. By Pe75, V.3.6], $\widetilde{\mathcal{C}}$ is an abelian category. The monomorphisms in $\widetilde{\mathcal{C}}$ are exactly the closed immersions; the epimorphisms are exactly the faithfully flat morphisms (see Pe75, V.3.2, V.3.4]). Also, note that the quotient morphism $G \rightarrow G / H$ is an fpqc torsor for any $G \in \widetilde{\mathcal{C}}$ and any subgroup scheme $H \subset G$ (see [SGA3, IV.5.1.7.1]). Using fpqc descent, it follows that $\mathcal{C}$ is a Serre subcategory of $\widetilde{\mathcal{C}}$.

Every commutative affine group scheme is an object of $\widetilde{\mathcal{C}}$; these objects form a full subcategory $\widetilde{\mathcal{L}}$, which is again a Serre subcategory by [SGA3, VIB.9.2, VIB.11.17]. Likewise, the profinite group schemes form a Serre subcategory $\widetilde{\mathcal{F}}$ of $\widetilde{\mathcal{L}}$.

By the main theorem of Pe75], every quasi-compact group scheme $G$ (possibly non-commutative) is the filtered inverse limit of algebraic quotient groups $G_{i}$ with transition functions affine for large $i$; equivalently, $G$ is essentially affine in the 
sense of [EGA, IV.8.13.4]. We now record a slightly stronger version of this result in our commutative setting as follows.

Lemma 2.1. Let $G \in \widetilde{\mathcal{C}}$.

(i) For any epimorphism $f: G \rightarrow H$ in $\widetilde{\mathcal{C}}$, where $H$ is affine (resp., profinite), there exists an affine (resp., profinite) subgroup scheme $H^{\prime} \subset G$ such that the composition $H^{\prime} \rightarrow G \rightarrow H$ is an epimorphism.

(ii) There is an exact sequence in $\widetilde{\mathcal{C}}$

$$
0 \longrightarrow H \longrightarrow G \longrightarrow A \longrightarrow 0,
$$

where $H$ is affine and $A$ is an abelian variety.

(iii) $G$ is the filtered inverse limit of its quotients $G / H$, where $H$ runs over the affine subgroup schemes of $G$ such that $G / H$ is algebraic.

Proof. (i) We may choose an affine subgroup scheme $L \subset G$ such that $G / L$ is algebraic. Then $f$ induces an epimorphism $G / L \rightarrow H / f(L)$, and $H / f(L)$ is affine. By the lifting property for the pair $(\mathcal{C}, \mathcal{L})$ (see [Br18, Lem. 3.1]), there exists an affine subgroup scheme $K \subset G / L$ such that the composition $K \rightarrow G / L \rightarrow H / f(L)$ is an epimorphism. Consider the preimage $H^{\prime}$ of $K$ in $G$. Then $H^{\prime}$ is affine (as an extension of $K$ by $L$ ) and the composition $H^{\prime} \rightarrow G \rightarrow H$ is an epimorphism. This shows the assertion for affine quotients. The assertion for profinite quotients follows similarly from the lifting property for the pair $(\mathcal{C}, \mathcal{F})$, obtained in $\operatorname{Br} 15$, Thm. 1.1] (see also Lu17, Thm. 3.2]).

(ii) Consider the neutral component $G^{0}$ of $G$; this is a connected subgroup scheme and the quotient $G / G^{0}$ is pro-étale (see [Pe75, V.4.1]). Moreover, by [Pe75, V.4.3.1], there is an exact sequence in $\widetilde{\mathcal{C}}$

$$
0 \longrightarrow H^{\prime} \longrightarrow G^{0} \longrightarrow A \longrightarrow 0
$$

where $H^{\prime}$ is affine and $A$ is an abelian variety. In view of (i), there is a profinite subgroup scheme $H^{\prime \prime} \subset G$ such that the composition $H^{\prime \prime} \rightarrow G \rightarrow G / G^{0}$ is an epimorphism. Then $H:=H^{\prime}+H^{\prime \prime}$ is an affine subgroup scheme of $G$, and $G / H$ is a quotient of $A$, hence an abelian variety.

(iii) Let $H$ be as in (ii). Then the subgroup schemes $H^{\prime} \subset H$ such that $H / H^{\prime}$ is algebraic form a filtered inverse system, and their intersection is trivial. Moreover, for any such subgroup scheme $H^{\prime}$, the quotient $G / H^{\prime}$ is algebraic (as an extension of $A$ by $\left.H / H^{\prime}\right)$. This yields the assertion in view of [Pe75, II.3.1.1].

In particular, every commutative quasi-compact group scheme $G$ is a filtered inverse limit of algebraic quotient groups with affine transition morphisms.

We now consider the pro category $\operatorname{Pro}(\mathcal{C})$ : its objects (the pro-algebraic groups) are the filtered inverse systems of objects of $\mathcal{C}$, and the morphisms are defined by

$$
\operatorname{Hom}_{\operatorname{Pro}(\mathcal{C})}\left(\lim _{\leftarrow} G_{i}, \lim _{\leftarrow} H_{j}\right):=\lim _{\leftarrow, j \rightarrow, i} \lim _{\mathcal{C}} \operatorname{Hom}_{\mathcal{C}}\left(G_{i}, H_{j}\right) .
$$

Recall that $\operatorname{Pro}(\mathcal{C})$ is an abelian category having enough projectives; moreover, the natural functor

$$
F: \mathcal{C} \longrightarrow \operatorname{Pro}(\mathcal{C})
$$

yields an equivalence of $\mathcal{C}$ with the Serre subcategory $\mathcal{C}^{\prime}$ of $\operatorname{Pro}(\mathcal{C})$ consisting of artinian objects (see, e.g., Oo66, I.4] for these facts). Also, recall that for any object $X$ of $\operatorname{Pro}(\mathcal{C})$, the artinian quotients $X_{i}$ of $X$ form a filtered inverse system, and the resulting map $X \rightarrow \lim _{\leftarrow} X_{i}$ is an isomorphism (see, e.g., DG70, V.2.2]). 
As a consequence, the restriction of $F$ to $\mathcal{L}$ extends to an equivalence of categories $\widetilde{\mathcal{L}} \stackrel{\simeq}{\longrightarrow} \operatorname{Pro}(\mathcal{L}) ;$ also, note that $\operatorname{Pro}(\mathcal{L})$ is a Serre subcategory of $\operatorname{Pro}(\mathcal{C})$.

Denote by $\widetilde{\mathcal{C}}^{\prime}$ the full subcategory of $\operatorname{Pro}(\mathcal{C})$ consisting of those objects $X$ such that there exists an exact sequence in $\operatorname{Pro}(\mathcal{C})$

$$
0 \longrightarrow Y \longrightarrow X \longrightarrow A \longrightarrow 0,
$$

where $Y \in \operatorname{Pro}(\mathcal{L})$ and $A$ is (the image under $F$ of) an abelian variety.

Lemma 2.2. $\quad$ (i) The objects of $\widetilde{\mathcal{C}}^{\prime}$ are exactly the pro-algebraic groups that are isomorphic to essentially affine objects.

(ii) $\widetilde{\mathcal{C}}^{\prime}$ is a Serre subcatgory of $\operatorname{Pro}(\mathcal{C})$.

(iii) Sending each $G \in \widetilde{\mathcal{C}}$ to the filtered inverse system of its algebraic quotients extends to an exact functor $S: \widetilde{\mathcal{C}} \rightarrow \widetilde{\mathcal{C}}^{\prime}$, which is an equivalence of categories.

Proof. (i) Let $X \in \operatorname{Pro}(\mathcal{C})$ be an essentially affine object. Then there is an exact sequence in $\operatorname{Pro}(\mathcal{C})$

$$
0 \longrightarrow Y \longrightarrow X \longrightarrow Z \longrightarrow 0
$$

where $Y$ is affine and $Z$ is algebraic. Thus, $Z$ is an extension of an abelian variety by an affine group scheme. Hence so is $X$; it follows that $X$ is an object of $\widetilde{\mathcal{C}^{\prime}}$.

Conversely, every object of $\operatorname{Pro}(\mathcal{C})$ that lies in an extension (2.1) is isomorphic to the essentially affine object consisting of the inverse system of quotients $X / Y^{\prime}$, where $Y^{\prime}$ is a subobject of $Y$.

(ii) Let $X \in \widetilde{\mathcal{C}^{\prime}}$ and consider a subobject $X^{\prime}$ of $X$ in $\operatorname{Pro}(\mathcal{C})$. Then we have a commutative diagram with exact rows

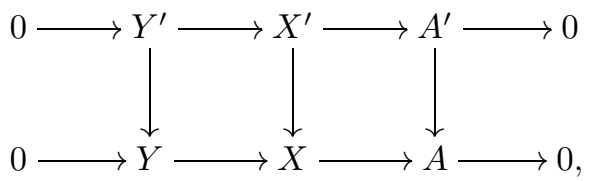

where the vertical arrows are monomorphisms. It follows that $Y^{\prime} \in \operatorname{Pro}(\mathcal{L})$, and $A^{\prime}$ is an extension of an abelian variety by a finite group scheme (Lemma 2.1). As a consequence, $X^{\prime} \in \widetilde{\mathcal{C}^{\prime}}$. Moreover, $X / X^{\prime}$ is an extension of $A / A^{\prime}$ by $Y / Y^{\prime}$, and hence is an object of $\widetilde{\mathcal{C}^{\prime}}$ as well. So $\widetilde{\mathcal{C}^{\prime}}$ is stable by subobjects and quotients. To show the stability by extensions, it suffices to check that $\widetilde{\mathcal{C}^{\prime}}$ contains all objects $X$ of $\operatorname{Pro}(\mathcal{C})$ which lie in an exact sequence

$$
0 \longrightarrow A \longrightarrow X \longrightarrow Y \longrightarrow 0,
$$

where $A$ is an abelian variety and $Y \in \operatorname{Pro}(\mathcal{L})$. But this follows from the fact that the pair $(\operatorname{Pro}(\mathcal{C}), \operatorname{Pro}(\mathcal{L}))$ satisfies the lifting property (see $[\operatorname{Br} 18$, Cor. 2.12]).

(iii) Note that $S(G)$ is essentially affine for any $G \in \widetilde{\mathcal{C}}$. Next, we define $S(f)$ for any morphism $f: G \rightarrow H$ in $\widetilde{\mathcal{C}}$. If $H \in \mathcal{C}$, then $f$ factors through a morphism $f^{\prime}: G^{\prime} \rightarrow H$ for any sufficiently large algebraic quotient $G^{\prime}$ of $G$; we then define $S(f)$ as the image of $f^{\prime}$ in

$$
\lim _{\rightarrow} \operatorname{Hom}_{\mathcal{C}}\left(G^{\prime}, H\right)=\operatorname{Hom}_{\operatorname{Pro}(\mathcal{C})}(S(G), S(H)) .
$$

This extends to an arbitrary $H \in \widetilde{\mathcal{C}}$ by using the equality

$$
\operatorname{Hom}_{\operatorname{Pro}(\mathcal{C})}\left(G, \lim _{\leftarrow} H^{\prime}\right)=\lim _{\leftarrow} \operatorname{Hom}_{\operatorname{Pro}(\mathcal{C})}\left(G, H^{\prime}\right) \text {. }
$$


To show that $S$ satisfies our assertions, recall from [EGA, IV.8.2.3] that for any essentially affine object $X=\left(X_{i}\right)$ of $\operatorname{Pro}(\mathcal{C})$, the inverse limit of the $X_{i}$ exists in $\widetilde{\mathcal{C}}$; denote this quasi-compact group scheme by $L(X)$. Moreover, $L$ extends to an equivalence from the full subcategory of $\operatorname{Pro}(\mathcal{C})$ consisting of the essentially affine objects, to the category $\widetilde{\mathcal{C}}$ (see [EGA, IV.8.13.5, IV.8.13.6]). By construction, $L \circ S$ is isomorphic to the identity functor of $\widetilde{\mathcal{C}}$. This yields the desired statement, except for the exactness of $S$. By [EGA, IV.8.13.6] again, $L$ commutes with products; equivalently, $L$ is additive. It follows that $S$ is additive as well. To show that it is exact, it suffices to check that $S$ preserves kernels and cokernels. In turn, it suffices to show that $S$ preserves monomorphisms and epimorphisms. But this follows readily from the stability of $\widetilde{\mathcal{C}}^{\prime}$ under subobjects and quotients.

Proposition 2.3. $\quad$ (i) For any object $G$ of $\widetilde{\mathcal{C}^{\prime}}$, the projective cover of $G$ in $\operatorname{Pro}(\mathcal{C})$ is an object of $\widetilde{\mathcal{C}^{\prime}}$ as well.

(ii) Every indecomposable projective of $\operatorname{Pro}(\mathcal{C})$ is an object of $\widetilde{\mathcal{C}}^{\prime}$.

(iii) $\widetilde{\mathcal{C}}$ has enough projectives.

Proof. (i) View $G$ as an extension of an abelian variety $A$ by an affine group scheme $H$. This readily yields an isomorphism of projective covers $P(G) \simeq P(H) \oplus P(A)$ with an obvious notation. Moreover, $P(H)$ is affine, and $P(A)$ is an extension of $A$ by an affine group scheme (see [Br18, Prop. 3.3]). Thus, $P(G) \in \widetilde{\mathcal{C}^{\prime}}$.

(ii) This follows from the fact that every indecomposable projective object of $\operatorname{Pro}(\mathcal{C})$ is the projective cover of an algebraic group (see, e.g., [DG70, V.2.4.3]).

(iii) By (i), the abelian category $\widetilde{\mathcal{C}}^{\prime}$ has enough projectives. Hence so does $\widetilde{\mathcal{C}}$ in view of Lemma 2.2 .

2.2. Universal affine covers of abelian varieties. Let $A$ be an abelian variety. By Proposition 2.3. $A$ has a projective cover $G_{A}$ in $\widetilde{\mathcal{C}}$. The resulting exact sequence,

$$
0 \longrightarrow H_{A} \longrightarrow G_{A} \stackrel{f_{A}}{\longrightarrow} A \longrightarrow 0,
$$

is the universal extension of $A$ by an affine group scheme, as follows from [Br18, §3.3] combined with Lemma 2.2. More specifically, for any commutative affine group scheme $H$, there is an isomorphism

$$
\operatorname{Hom}_{\widetilde{\mathcal{L}}}\left(H_{A}, H\right) \stackrel{\simeq}{\longrightarrow} \operatorname{Ext}_{\widetilde{\mathcal{C}}}^{1}(A, H)
$$

given by pushout of the extension (2.2). Also, by Lemma 2.2 again and $\mathrm{Br} 18, \S \S 2.3$, 3.4], the projective objects of $\widetilde{\mathcal{C}}$ are exactly the products $P \times G_{B}$, where $P$ is a projective object of $\widetilde{\mathcal{L}}$ and $B$ is an abelian variety.

Note that $f_{A}: G_{A} \rightarrow A$ is the filtered inverse limit of all anti-affine extensions of $A$, i.e., of all algebraic groups $G$ equipped with an epimorphism $f: G \rightarrow A$ such that $\operatorname{Ker}(f)$ is affine and $\mathcal{O}(G)=k$ (see [Br18, Lem. 2.14]). In particular, $\mathcal{O}\left(G_{A}\right)=k$ as well. Using [Pe75, 4.2.2], it follows that $G_{A}$ is geometrically integral.

Next, we show that (2.2) is the universal extension of $A$ by a (possibly noncommutative) affine group scheme.

Theorem 2.4. Let $G$ be a quasi-compact group scheme, and let $f: G \rightarrow A$ be a faithfully flat morphism of group schemes with affine kernel $H$. Then there exist unique morphisms of group schemes $\varphi: G_{A} \rightarrow G, \psi: H_{A} \rightarrow H$ such that we have 
a commutative diagram with exact rows

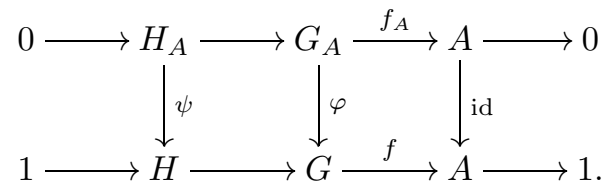

Moreover, $\varphi$ factors through the center of the neutral component $G^{0}$.

Proof. As $G$ is quasi-compact, we may freely use the results of [Pe75, V.3] on the representability of the fpqc quotients of $G$ and its subgroup schemes. Also, recall the affinization theorem (see [Pe75, 4.2.2]): $G$ has a largest normal subgroup scheme $N$ such that $G / N$ is affine; moreover, $N$ is geometrically integral and contained in the center of $G^{0}$. In particular, $N$ is an object of $\widetilde{\mathcal{C}}$. Let $B$ denote the schemetheoretic image of $N$ under $f: G \rightarrow A$. Then $B$ is an abelian subvariety of $A$; moreover, $A / B$ is isomorphic to a quotient of $G / N$, and hence is affine. So $B=A$, and hence $f$ restricts to an epimorphism $g: N \rightarrow A$ in $\widetilde{\mathcal{C}}$ with affine kernel. This readily yields the existence of $\varphi, \psi$.

For the uniqueness, just note that $\varphi$ factors through $N$, since every affine quotient of $G_{A}$ is trivial.

Proposition 2.5. The formation of $G_{A}$ commutes with base change under arbitrary field extensions.

Proof. Let $k^{\prime} / k$ be a field extension. For any anti-affine extension $f: G \rightarrow A$, the morphism obtained by base change $f_{k^{\prime}}: G_{k^{\prime}} \rightarrow A_{k^{\prime}}$ is an anti-affine extension again, as $\mathcal{O}\left(G_{k^{\prime}}\right)=\mathcal{O}(G) \otimes_{k} k^{\prime}$ (see, e.g., [SGA3, VIB.11.1]). Also, since $G_{A}=$ $\lim _{\leftarrow} G$ (where $G$ runs over the above anti-affine extensions of $A$ ) and base change commutes with filtered inverse limits, we obtain $\left(G_{A}\right)_{k^{\prime}}=\lim _{\leftarrow} G_{k^{\prime}}$. This yields a commutative diagram with exact rows

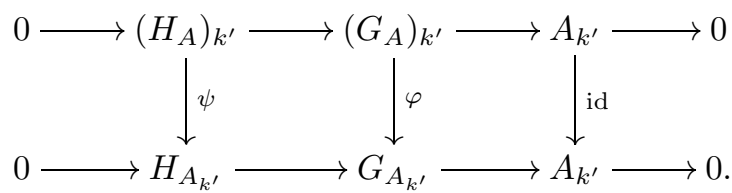

As a consequence, $\operatorname{Coker}(\varphi) \simeq \operatorname{Coker}(\psi)$ is affine. Since $\left(G_{A}\right)_{k^{\prime}}$ is anti-affine, it follows that $\varphi$ is an epimorphism. As $G_{A_{k^{\prime}}}$ is projective in $\widetilde{\mathcal{C}}_{k^{\prime}}$, this yields an isomorphism $\left(G_{A}\right)_{k^{\prime}} \simeq G_{A_{k^{\prime}}} \times \operatorname{Ker}(\varphi)$. In particular, $\operatorname{Ker}(\varphi)$ is a quotient of $\left(G_{A}\right)_{k^{\prime}}$. $\operatorname{But} \operatorname{Ker}(\varphi) \simeq \operatorname{Ker}(\psi)$ is affine, and hence trivial.

The commutative affine group scheme $H_{A}$ lies in a unique exact sequence

$$
0 \longrightarrow M_{A} \longrightarrow H_{A} \longrightarrow U_{A} \longrightarrow 0,
$$

where $M_{A}$ is of multiplicative type and $U_{A}$ is unipotent; when $k$ is perfect, this exact sequence has a unique splitting (see [DG70, IV.3.1.1]). We now describe the structure of $M_{A}$.

Lemma 2.6. $\quad$ (i) For any field extension $k^{\prime} / k$, there is a natural isomorphism $\operatorname{Hom}_{\widetilde{\mathcal{L}}}\left(H_{A_{k^{\prime}}}, \mathbb{G}_{m, k^{\prime}}\right) \simeq \widehat{A}\left(k^{\prime}\right)$.

(ii) The Cartier dual of $M_{A}$ is the $\Gamma$-module $\widehat{A}(\bar{k})$. 
(iii) The group scheme $H_{A}$ is not algebraic. When $k$ is imperfect, the exact sequence (2.4) does not split.

Proof. (i) By Proposition 2.5 we may assume that $k^{\prime}=k$. Then (2.3) yields a natural isomorphism $\operatorname{Hom}_{\widetilde{\mathcal{L}}}\left(H_{A}, \mathbb{G}_{m}\right) \simeq \operatorname{Ext}_{\widetilde{\mathcal{C}}}^{1}\left(A, \mathbb{G}_{m}\right)$, which implies the asssertion in view of the Weil-Barsotti formula (see, e.g., Oo66, III.17, III.18]).

(ii) This follows readily from (i) by taking $k^{\prime}=\bar{k}$.

(iii) Assume that $H_{A}$ is an algebraic group. Then $\operatorname{Hom}_{\widetilde{\mathcal{L}}}\left(H_{A_{\bar{k}}}, \mathbb{G}_{m, \bar{k}}\right)$ is a finitely generated abelian group. But $\widehat{A}(\bar{k})$ is not finitely generated, since it has non-zero $\ell$-torsion for any prime $\ell \neq p$; a contradiction.

Next, assume that the extension (2.4) splits. Then the resulting isomorphism $H_{A} \simeq M_{A} \times U_{A}$ and Proposition 2.5 yield an isomorphism

$$
\operatorname{Hom}_{\widetilde{\mathcal{L}}}\left(H_{A_{k^{\prime}}}, \mathbb{G}_{m, k^{\prime}}\right) \simeq \operatorname{Hom}_{\widetilde{\mathcal{L}}}\left(\left(M_{A}\right)_{k^{\prime}}, \mathbb{G}_{m, k^{\prime}}\right)
$$

for any field extension $k^{\prime} / k$. As $M_{A}$ is of multiplicative type, it follows that the natural map $\operatorname{Hom}_{\tilde{\mathcal{L}}}\left(H_{A_{k_{s}}}, \mathbb{G}_{m, k_{s}}\right) \rightarrow \operatorname{Hom}_{\tilde{\mathcal{L}}}\left(H_{A_{\bar{k}}}, \mathbb{G}_{m, \bar{k}}\right)$ is an isomorphism. In view of (i), this yields the equality $\widehat{A}\left(k_{s}\right)=\widehat{A}(\bar{k})$. Choose a non-empty open affine subscheme $U$ of $\widehat{A}$; then $U\left(k_{s}\right)=U(\bar{k})$ as well. By Noether normalization, there exists a finite surjective morphism $F: U \rightarrow \mathbb{A}_{k}^{g}$. If $k$ is imperfect, we may choose $t \in \bar{k} \backslash k_{s}$; then the fiber of $F$ at $(t, 0, \ldots, 0) \in \mathbb{A}_{k}^{g}(\bar{k})$ contains no $k_{s}$-rational point, a contradiction.

We now turn to the structure of $U_{A}$. If $p=0$, then $U_{A}$ is the vector group associated with the dual vector space of $\mathrm{H}^{1}\left(A, \mathcal{O}_{A}\right)$ (see Br18, Lem. 3.8]); thus, $U_{A} \simeq\left(\mathbb{G}_{a}\right)^{g}$. If $p>0$, then $U_{A}$ is profinite; more specifically, $U_{A}$ is the largest unipotent quotient of the profinite fundamental group of $A, \lim _{\leftarrow} A[n]$ (as follows from $\left[\mathrm{Br} 18\right.$, Thm. 3.10]). Thus, $U_{A}$ is the largest unipotent quotient of its pro- $p$ part, $A\left[p^{\infty}\right]:=\lim _{\leftarrow} A\left[p^{n}\right]$.

Next, recall from [Mu08, §15] that

$$
A\left[p^{n}\right]_{\bar{k}} \simeq\left(\mathbb{Z} / p^{n} \mathbb{Z}\right)_{\bar{k}}^{\frac{r}{k}} \times\left(\mu_{p^{n}}\right)_{\bar{k}}^{\frac{r}{k}} \times G_{n},
$$

where $r=r_{A}$ is an integer independent of $n$ (called the $p$-rank of $A$ ), and $G_{n}$ is a unipotent infinitesimal $\bar{k}$-group scheme of order $p^{2 n(g-r)}$. The abelian variety $A$ is said to be ordinary if it has maximal $p$-rank, i.e., $r=g$ (see [ST16, §2.1] for further characterizations of ordinary abelian varieties). It follows that

$$
\left(U_{A}\right)_{\bar{k}} \simeq \lim _{\leftarrow}\left(\mathbb{Z} / p^{n} \mathbb{Z}\right)_{\bar{k}}^{r} \times G_{n} n \simeq\left(\mathbb{Z}_{p}\right)_{\bar{k}}^{r} \times \lim _{\leftarrow} G_{n} .
$$

As a consequence, $A$ is ordinary if and only if $U_{A}$ is pro-étale; then

$$
U_{A}\left(k_{s}\right) \simeq\left(\mathbb{Z}_{p}\right)^{g}
$$

Remark 2.7. When $k$ is algebraically closed, the groups $\operatorname{Ext}_{\mathcal{C}}^{1}(A, H)$, where $H$ is a commutative affine algebraic group, have been determined by $\mathrm{Wu}$ in [Wu86. His results may be recovered from the above description of $H_{A}$ in view of the isomorphism (2.3).

Also, this description can be interpreted in terms of formal groups associated with $\widehat{A}$, via Cartier duality which yields an anti-equivalence of $\widetilde{\mathcal{L}}$ with the category of commutative formal $k$-groups (see [SGA3, VIIB.2], that we will freely use as a 
general reference for formal groups). Under that equivalence, the exact sequence (2.4) corresponds to an exact sequence of formal groups,

$$
0 \longrightarrow \mathrm{D}\left(U_{A}\right) \longrightarrow \mathrm{D}\left(H_{A}\right) \longrightarrow \mathrm{D}\left(M_{A}\right) \longrightarrow 0,
$$

where $\mathrm{D}\left(U_{A}\right)$ is infinitesimal and $\mathrm{D}\left(M_{A}\right)$ is étale; this exact sequence splits if and only if $k$ is perfect. By Lemma 2.6(ii), $\mathrm{D}\left(M_{A}\right)$ corresponds to the $\Gamma$-group $\widehat{A}(\bar{k})$ under the equivalence of étale formal groups with $\Gamma$-groups.

Also, if $p=0$, then $\mathrm{D}\left(U_{A}\right)$ is the infinitesimal formal group associated with the commutative Lie algebra $\mathrm{H}^{1}\left(A, \mathcal{O}_{A}\right)$. Since the latter is the Lie algebra of $\widehat{A}$, we may identify $\mathrm{D}\left(U_{A}\right)$ with the infinitesimal formal neighborhood of the origin in $\widehat{A}$.

This still holds if $p>0$; then the above infinitesimal formal group is isomorphic to $\lim _{\rightarrow} \operatorname{Ker}\left(\mathrm{F}_{\widehat{A}}^{n}\right)$, where $\mathrm{F}_{\widehat{A}}^{n}: \widehat{A} \rightarrow \widehat{A}^{\left(p^{n}\right)}$ denotes the $n$th relative Frobenius morphism. Moreover, we have canonical isomorphisms

$$
\mathrm{D}\left(\lim _{\rightarrow} \operatorname{Ker}\left(\mathrm{F}_{\widehat{A}}^{n}\right)\right) \simeq \lim _{\leftarrow} \mathrm{D}\left(\operatorname{Ker}\left(\mathrm{F}_{\widehat{A}}^{n}\right)\right) \simeq \lim _{\leftarrow} \operatorname{Ker}\left(\mathrm{V}_{A}^{n}\right),
$$

where $\mathrm{V}_{A}^{n}: A^{\left(p^{n}\right)} \rightarrow A$ denotes the $n$th Verschiebung, and the second isomorphism follows from duality between Frobenius and Verschiebung. Also, we have a canonical exact sequence

$$
0 \longrightarrow \operatorname{Ker}\left(\mathrm{F}_{A}^{n}\right) \longrightarrow A\left[p^{n}\right] \longrightarrow \operatorname{Ker}\left(\mathrm{V}_{A}^{n}\right) \longrightarrow 0
$$

for any $n \geq 1$; as a consequence, $\lim _{\leftarrow} \operatorname{Ker}\left(\mathrm{V}_{A}^{n}\right)$ may be identified with the largest unipotent quotient of $A\left[p^{\infty}\right]$, i.e., with $U_{A}$.

2.3. Applications to homogeneous vector bundles. Let $\pi: E \rightarrow A$ be a vector bundle. For any scheme $S$, consider the set $G(S)$ consisting of the pairs $(\varphi, a)$ satisfying the following conditions:

(i) $\varphi: E_{S} \rightarrow E_{S}$ is an isomorphism of $S$-schemes, and $a \in A(S)$.

(ii) The diagram

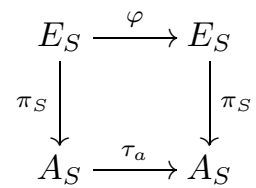

commutes, where $\tau_{a}$ denotes the translation by $a$ in $A_{S}$.

(iii) The isomorphism of $A_{S}$-schemes $E_{S} \rightarrow \tau_{a}^{*}\left(E_{S}\right)$ induced by $\varphi$, is an isomorphism of vector bundles.

Alternatively, we may view $G(S)$ either as the set of pairs $(a, \psi)$, where $a \in A(S)$ and $\psi: E_{S} \rightarrow \tau_{a}^{*}\left(E_{S}\right)$ is an isomorphism of vector bundles (see [Mi73, p. 72]), or as the group of automorphisms of the $S$-scheme $E_{S}$ which lift translations in $A_{S}$ and commute with the action of $\mathbb{G}_{m, S}$ by multiplication on fibers.

Clearly, $G(S)$ is a group for componentwise multiplication of pairs $(\varphi, a)$; moreover, the assignment $S \mapsto G(S)$ extends to a group functor that we still denote by $G$, or $G_{E}$ to emphasize its dependence in $E$. The second projection yields a morphism of group functors $f: G \rightarrow A$; the kernel of $f$ is the group functor $H=H_{E}$ of automorphisms of the vector bundle $E$.

Lemma 2.8. $\quad$ (i) $H$ is a smooth connected affine algebraic group.

(ii) $G$ is an algebraic group.

(iii) $E$ is equipped with a G-linearization. 
Proof. (i) Note that $H$ is the group of invertibles of the monoid functor of endomorphisms of the vector bundle $E$, and this monoid functor is represented by an affine space. So the assertion follows from DG70, II.2.3.6].

(ii) The group functor of $\mathbb{G}_{m}$-equivariant automorphisms of $E$ is represented by a group scheme $\operatorname{Aut}_{E}^{\mathbb{G}_{m}}$, locally of finite type; moreover, we have an exact sequence of group schemes

$$
1 \longrightarrow H \longrightarrow \operatorname{Aut}_{E}^{\mathbb{G}_{m}} \longrightarrow \operatorname{Aut}_{A}
$$

(see [BSU13, Prop. 6.3.2]). Viewing $A$ as a subgroup scheme of Aut $_{A}$ via its action by translation, we may identify $G$ with the pullback of $A$ in $\operatorname{Aut}_{E}^{\mathbb{G}_{m}}$; this yields the assertion.

(iii) This follows, e.g., from [SGA3, I.6.5.3].

By Lemma 2.8, there is an exact sequence of algebraic groups

$$
1 \longrightarrow H_{E} \longrightarrow G_{E} \stackrel{f}{\longrightarrow} A .
$$

We say that $E$ is homogeneous if $f$ is faithfully flat; equivalently, (2.7) is right exact. Since $f$ is a morphism of algebraic groups and $A$ is smooth, this amounts to the following condition: for any $a \in A(\bar{k})$, the translation by $a$ in $A_{\bar{k}}$ lifts to an automorphism of the $\bar{k}$-variety $E_{\bar{k}}$, linear on fibers. Equivalently, $E_{\bar{k}} \simeq \tau_{a}^{*}\left(E_{\bar{k}}\right)$ for any $a \in A(\bar{k})$.

As a consequence, if $E$ is homogeneous, then so is the dual vector bundle $E^{\vee}$; if in addition $F$ is a homogeneous vector bundle over $A$, then both $E \oplus F$ and $E \otimes F$ are homogeneous as well. Thus, the homogeneous vector bundles form a full subcategory of the category $\mathrm{Vec}_{A}$ of vector bundles over $A$, stable under finite direct sums, duals, and tensor products. We denote this additive tensor subcategory by $\mathrm{HVec}_{A}$.

Let $E$ be a homogeneous vector bundle over $A$. Then the algebraic group $G_{E}$ is smooth and connected, since so are $H_{E}$ and $A$. This group acts transitively on $A$ via $f$, and the stabilizer of the origin 0 equals $H_{E}$. The $G_{E}$-linearization of $E$ restricts to an action of $H_{E}$ on the fiber $E_{0}$ via a representation

$$
\rho: H_{E} \longrightarrow \mathrm{GL}\left(E_{0}\right) \text {. }
$$

Moreover, the morphism $G_{E} \times E_{0} \rightarrow E$ given by the action, factors through an isomorphism

$$
G_{E} \times{ }^{H_{E}} E_{0} \stackrel{\simeq}{\longrightarrow} E,
$$

where the left-hand side denotes the quotient of $G_{E} \times E_{0}$ by the action of $H_{E}$ via $h \cdot(g, x):=\left(g h^{-1}, \rho(h) x\right)$; this is the vector bundle over $A$ associated with the $H_{E}$-torsor $G_{E} \rightarrow A$ and the $H_{E}$-representation in $E_{0}$.

Also, by Theorem 2.4, we have a commutative diagram with exact rows

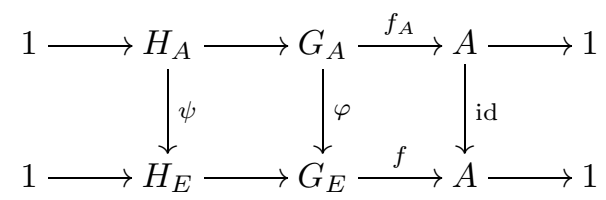

for unique morphisms $\varphi=\varphi_{E}, \psi=\psi_{E}$; moreover, $\varphi_{E}$ factors through the center of $G_{E}$. This yields a representation $\rho \circ \psi: H_{A} \rightarrow \operatorname{GL}\left(E_{0}\right)$ and an isomorphim $G_{A} \times{ }^{H_{A}} E_{0} \simeq E$. In particular, $E$ is $G_{A}$-linearized. 
Conversely, given a finite-dimensional representation $\rho: H_{A} \rightarrow \mathrm{GL}(V)$, the quotient $H:=H_{A} / \operatorname{Ker}(\rho)$ is algebraic and lies in an exact sequence

$$
0 \longrightarrow H \longrightarrow G \longrightarrow A \longrightarrow 0,
$$

where $G:=G_{A} / \operatorname{Ker}(\rho)$ is algebraic as well. Thus, we may form the associated vector bundle $\mathcal{L}_{G / H}(V)$ (see [Ja03, I.5.8, I.5.15]): this is a $G$-linearized vector bundle on $A$, and hence is homogeneous. We denote this vector bundle by

$$
\mathcal{L}_{A}(V)=G_{A} \times{ }^{H_{A}} V \longrightarrow A .
$$

Its fiber at 0 is $V$, on which $H_{A}$ acts via the representation $\rho$.

Denote by $H_{A}$-mod the category of finite-dimensional representations of $H_{A}$; this is an abelian tensor category. We may now state the following.

Theorem 2.9. The above assignments $E \mapsto E_{0}, V \mapsto G_{A} \times{ }^{H_{A}} V$ extend to exact functors

$$
\mathcal{M}_{A}: \mathrm{HVec}_{A} \longrightarrow H_{A} \text {-mod, } \mathcal{L}_{A}: H_{A} \text {-mod } \longrightarrow \mathrm{HVec}_{A},
$$

which are quasi-inverse equivalences of additive tensor categories.

Proof. Let $E, F$ be homogeneous vector bundles over $A$. Since they both are $G_{A^{-}}$ linearized, the finite-dimensional vector space $\operatorname{Hom}_{\mathrm{Vec}_{A}}(E, F)$ (consisting of the morphisms of vector bundles $\gamma: E \rightarrow F)$ is equipped with a linear representation of $G_{A}$ (see [SGA3, I.6.6.2]). As $\mathcal{O}\left(G_{A}\right)=k$, this representation is trivial, i.e., every $\gamma$ as above is $G_{A}$-equivariant. As a consequence, the restriction $\gamma_{0}: E_{0} \rightarrow F_{0}$ is $H_{A}$-equivariant, where $H_{A}$ acts on $E_{0}$ and $F_{0}$ via the above representations. The assignment $\gamma \mapsto \gamma_{0}$ defines the functor $\mathcal{M}_{A}$ on morphisms.

Given $E, F$ as above, consider the image $G$ of the product morphism

$$
\left(\varphi_{E}, \varphi_{F}\right): G_{A} \longrightarrow G_{E} \times G_{F} .
$$

Then $G$ is an algebraic quotient of $G_{A}$, and lies in an exact sequence of algebraic groups

$$
0 \longrightarrow H \longrightarrow G \longrightarrow A \longrightarrow 0
$$

for some algebraic quotient $H$ of $H_{A}$. Moreover, $E \simeq G \times{ }^{H} E_{0}, F \simeq G \times{ }^{H} F_{0}$, and $G$ acts trivially on $\operatorname{Hom}_{\operatorname{Vec}_{A}}(E, F)$. This yields an isomorphism

$$
\operatorname{Hom}_{\operatorname{Vec}_{A}}(E, F) \simeq \operatorname{Hom}_{\operatorname{Vec}_{A}}^{G}\left(G \times{ }^{H} E_{0}, G \times{ }^{H} F_{0}\right) .
$$

The right-hand side is contained in the set $\operatorname{Hom}^{G}\left(G \times{ }^{H} E_{0}, G \times{ }^{H} F_{0}\right)$ of $G$ equivariant morphisms of schemes, which is identified with $\operatorname{Hom}^{H}\left(E_{0}, G \times{ }^{H} F_{0}\right)$ via restriction; this identifies the subset $\operatorname{Hom}_{\mathrm{Vec}_{A}}^{G}\left(G \times{ }^{H} E_{0}, G \times{ }^{H} F_{0}\right)$ with the subset of $H$-equivariant linear maps $E_{0} \rightarrow F_{0}$. As a consequence, the functor $\mathcal{M}_{A}$ is fully faithful. It is essentially surjective, since $\mathcal{M}_{A}\left(\mathcal{L}_{A}(V)\right)=V$ for any finite-dimensional $H_{A}$-module $V$.

Next, note that every morphism of $H_{A}$-modules $u: V \rightarrow W$ yields a morphism of associated vector bundles $\mathcal{L}_{A}(u): \mathcal{L}_{A}(V) \rightarrow \mathcal{L}_{A}(W)$ such that $\mathcal{L}_{A}(u)_{0}=u$, as may be checked by factoring both representations $H_{A} \rightarrow \mathrm{GL}(V), H_{A} \rightarrow \mathrm{GL}(W)$ through a common algebraic quotient of $H_{A}$. This defines the functor $\mathcal{L}_{A}$ on morphisms, and shows that it is quasi-inverse to $\mathcal{M}_{A}$. Clearly, $\mathcal{L}_{A}$ and $\mathcal{M}_{A}$ are exact and preserve finite direct sums, duals, and tensor products.

Corollary 2.10. The subcategory $\mathrm{HVec}_{A}$ of $\mathrm{Vec}_{A}$ is abelian and stable under direct summands. 
Next, we obtain a key vanishing result for the coherent cohomology of $G_{A}$.

Proposition 2.11. $\mathrm{H}^{i}\left(G_{A}, \mathcal{O}_{G_{A}}\right)=0$ for any $i \geq 1$.

Proof. To simplify the notation, we set $G:=G_{A}, H:=H_{A}$, and $f:=f_{A}$. Since the morphism $f: G \rightarrow A$ is affine, we have $R^{i} f_{*}\left(\mathcal{O}_{G}\right)=0$ for all $i \geq 1$ in view of [EGA, III.1.3.2]. This yields isomorphisms

$$
\mathrm{H}^{i}\left(G, \mathcal{O}_{G}\right) \cong \mathrm{H}^{i}\left(A, f_{*}\left(\mathcal{O}_{G}\right)\right), \quad(i \geq 0) .
$$

Next, recall that $G \simeq \lim _{\leftarrow} G^{\prime}$, where the limit is taken over the filtered inverse system of anti-affine extensions $f^{\prime}: G^{\prime} \rightarrow A$. Thus, we have an isomorphism of quasi-coherent sheaves of $\mathcal{O}_{A}$-algebras

$$
f_{*}\left(\mathcal{O}_{G}\right) \simeq \lim _{\rightarrow} f_{*}^{\prime}\left(\mathcal{O}_{G^{\prime}}\right) .
$$

Since cohomology commutes with direct limits, this yields in turn isomorphisms

$$
\mathrm{H}^{i}\left(G, \mathcal{O}_{G}\right) \simeq \lim _{\rightarrow} \mathrm{H}^{i}\left(A, f_{*}^{\prime}\left(\mathcal{O}_{G^{\prime}}\right)\right) \simeq \lim _{\rightarrow} \mathrm{H}^{i}\left(G^{\prime}, \mathcal{O}_{G^{\prime}}\right), \quad(i \geq 0)
$$

As each $G^{\prime}$ is anti-affine, the coherent cohomology ring $\mathrm{H}^{*}\left(G^{\prime}, \mathcal{O}_{G^{\prime}}\right)$ is the exterior algebra over $\mathrm{H}^{1}\left(G^{\prime}, \mathcal{O}_{G^{\prime}}\right)$ (see $\mathrm{Br13}$, Thm. 1.1]). As a consequence, $\mathrm{H}^{*}\left(G, \mathcal{O}_{G}\right)$ is the exterior algebra over $\mathrm{H}^{1}\left(G, \mathcal{O}_{G}\right)$. Thus, it suffices to show the vanishing of $\mathrm{H}^{1}\left(G, \mathcal{O}_{G}\right)$. For this, we may assume that $k$ is algebraically closed, in view of Proposition 2.5.

By adapting the argument of [To13, Lem. 9.2] (see also [Se97, Thm. VII.5]), one may check that the canonical map

$$
\operatorname{Ext}_{\mathcal{C}}^{1}\left(G^{\prime}, \mathbb{G}_{a}\right) \longrightarrow \mathrm{H}^{1}\left(G^{\prime}, \mathcal{O}_{G^{\prime}}\right)^{G^{\prime}}
$$

is an isomorphism, where the right-hand side denotes the subspace of $G^{\prime}$-invariants in $\mathrm{H}^{1}\left(G^{\prime}, \mathcal{O}_{G^{\prime}}\right)$. As $\mathrm{H}^{1}\left(G^{\prime}, \mathcal{O}_{G^{\prime}}\right)$ is a (rational) $G^{\prime}$-module (see, e.g., [Br13, Lem. 2.1]) and $G^{\prime}$ is anti-affine, this yields compatible isomorphisms

$$
\operatorname{Ext}_{\mathcal{C}}^{1}\left(G^{\prime}, \mathbb{G}_{a}\right) \simeq \mathrm{H}^{1}\left(G^{\prime}, \mathcal{O}_{G^{\prime}}\right),
$$

and hence an isomorphism

$$
\operatorname{Ext}_{\operatorname{Pro}(\mathcal{C})}^{1}\left(G, \mathbb{G}_{a}\right) \simeq \mathrm{H}^{1}\left(G, \mathcal{O}_{G}\right),
$$

by using [DG70, V.2.3.9]. The desired vanishing follows from this, since $G$ is projective in $\operatorname{Pro}(\mathcal{C})$.

Alternatively, the vanishing of $\mathrm{H}^{1}\left(G, \mathcal{O}_{G}\right)$ can be obtained as follows. Let again $f^{\prime}: G^{\prime} \rightarrow A^{\prime}$ be an anti-affine extension and consider the Albanese morphism $\alpha: G^{\prime} \rightarrow A^{\prime}$; then $f^{\prime}$ is the composition of $\alpha$ with an isogeny $A^{\prime} \rightarrow A$. If $p=0$ and $\alpha$ lifts to an epimorphism $G^{\prime} \rightarrow E\left(A^{\prime}\right)$ (the universal vector extension of $A^{\prime}$ ), then $\mathrm{H}^{1}\left(G^{\prime}, \mathcal{O}_{G^{\prime}}\right)=0$ by [Br13, Prop. 4.1, Prop. 4.3]; this yields the desired vanishing. On the other hand, if $p>0$, then the pullback $\alpha^{*}: \mathrm{H}^{*}\left(A^{\prime}, \mathcal{O}_{A^{\prime}}\right) \rightarrow \mathrm{H}^{*}\left(G^{\prime}, \mathcal{O}_{G^{\prime}}\right)$ is an isomorphism by Br13, Cor. 4.2]. The commutative diagram

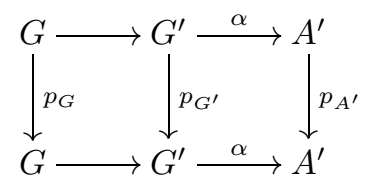


where the vertical arrows are multiplication maps, yields a commutative diagram of pullbacks

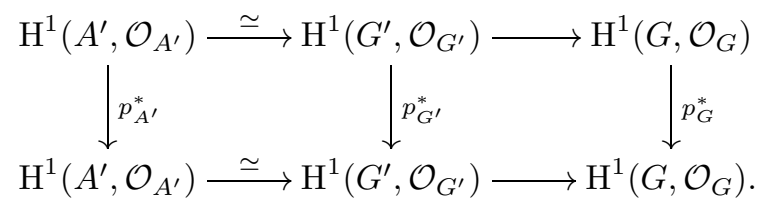

Moreover, $p_{G}$ is an isomorphism by $\mathrm{Br} 18$, Lem. 3.4]; hence so is $p_{G}^{*}$. Thus, it suffices to show that $p_{A^{\prime}}^{*}=0$. But this follows from the isomorphism $\operatorname{Ext}_{\mathcal{C}}^{1}\left(A^{\prime}, \mathbb{G}_{a}\right) \cong$ $\mathrm{H}^{1}\left(A^{\prime}, \mathcal{O}_{A^{\prime}}\right)$ (see [Se97, Thm. VII.5]) together with the equality $p_{A^{\prime}}^{*}=\left(p_{\mathbb{G}_{a}}\right)_{*}=0$ in $\operatorname{Ext}_{\mathcal{C}}^{1}\left(A^{\prime}, \mathbb{G}_{a}\right)$.

Theorem 2.12. Let $V, W$ be finite-dimensional $H_{A}$-modules. Then the map

$$
\operatorname{Ext}_{H_{A}}^{i}(V, W) \longrightarrow \operatorname{Ext}_{A}^{i}\left(\mathcal{L}_{A}(V), \mathcal{L}_{A}(W)\right)
$$

is an isomorphism for any $i \geq 0$, where the right-hand side denotes the higher extension group of coherent sheaves on $A$.

Proof. Denoting by $V^{\vee}$ the dual $H_{A}$-module of $V$, the statement can be reformulated as follows: the map

$$
\mathrm{H}^{i}\left(H_{A}, V^{\vee} \otimes_{k} W\right) \longrightarrow \mathrm{H}^{i}\left(A, \mathcal{L}_{A}\left(V^{\vee} \otimes_{k} W\right)\right)
$$

is an isomorphism for any $i \geq 0$ (use [Ja03, I.4.2, I.4.4] and the compatibility of $\mathcal{L}_{A}$ with duals and tensor products). This holds for $i=0$ by Theorem 2.9. Thus, it suffices to extend $\mathcal{L}_{A}$ to an exact functor from $H_{A}$-modules to quasi-coherent sheaves on $A$, which takes injective modules to acyclic sheaves; then the statement will follow from a degenerate case of Grothendieck's spectral sequence.

We first check that $\mathcal{L}_{A}$ extends uniquely to an exact functor, commuting with filtered direct limits, from the category $H_{A}$-Mod of all $H_{A}$-modules (not necessarily finite-dimensional), to the category $\mathrm{QCoh}_{A}$ of quasi-coherent sheaves on $A$. Indeed, $H_{A}$-Mod is a Grothendieck category and its noetherian objects are exactly those of $H_{A}$-mod; as a consequence, $H_{A}$-Mod is equivalent to the ind category $\operatorname{Ind}\left(H_{A}\right.$-mod $)$. Likewise, $\mathrm{QCoh}_{A}$ is equivalent to $\operatorname{Ind}\left(\mathrm{Coh}_{A}\right)$. So the desired assertion follows from [KS05, 6.1.9, 8.6.8].

We still denote the extended functor by

$$
\mathcal{L}_{A}: H_{A} \text {-Mod } \longrightarrow \mathrm{QCoh}_{A} \text {. }
$$

We now check that $\mathcal{L}_{A}$ takes injectives to acyclics. By [Ja03, I.3.10], every injective $H_{A}$-module is a direct summand of a direct sum of copies of $\mathcal{O}\left(H_{A}\right)$; hence it suffices to show that $\mathrm{H}^{i}\left(A, \mathcal{L}_{A}\left(\mathcal{O}\left(H_{A}\right)\right)\right)=0$ for all $i \geq 1$. In view of (the proof of) Proposition 2.11, it suffices in turn to check that

$$
\mathcal{L}_{A}\left(\mathcal{O}\left(H_{A}\right)\right) \simeq f_{*}\left(\mathcal{O}_{G_{A}}\right)
$$

where $H_{A}$ acts on $\mathcal{O}\left(H_{A}\right)$ via the regular representation. Recall that $\mathcal{O}\left(H_{A}\right)=$ $\lim _{\leftarrow} \mathcal{O}(H)$ and $f_{*}\left(\mathcal{O}_{G_{A}}\right)=\lim _{\rightarrow} f_{*}^{\prime}\left(\mathcal{O}_{G}\right)$, where both limits run over all anti-affine extensions $0 \longrightarrow H \longrightarrow G \stackrel{f}{\longrightarrow} A \longrightarrow 0$. Also, recall that $\mathcal{L}_{A}(V)$ is defined as the associated sheaf $\mathcal{L}_{G / H}(V)$ for any finite-dimensional $H$-module $V$, and hence for any $H$-module. By [Ja03, I.5.18], there are compatible isomorphisms

$$
\mathcal{L}_{A}(\mathcal{O}(H)) \stackrel{\simeq}{\longrightarrow} f_{*}^{\prime}\left(\mathcal{O}_{G}\right) .
$$

This yields the desired isomorphism (2.8). 
Corollary 2.13. The subcategory $\mathrm{HVec}_{A}$ of $\mathrm{Vec}_{A}$ is stable under extensions.

\section{Representations of COMmutative AFFine GRoup sChemes}

Throughout this section, we consider linear representations of a fixed commutative affine $k$-group scheme $H$. We use the book [Ja03] as a general reference for representation theory.

3.1. Irreducible representations. The aim of this subsection is to construct the irreducible representations of $H$ (Proposition [3.1), and classify them up to equivalence (Proposition 3.3).

Define a character of $H$ as a morphism of $\bar{k}$-group schemes

$$
\chi: H_{\bar{k}} \longrightarrow \mathbb{G}_{m, \bar{k}} \text {. }
$$

The characters form an abelian group that we denote by $\mathrm{X}(H)$. Every such character $\chi$ may be viewed as an element of $\mathcal{O}\left(H_{\bar{k}}\right)=\mathcal{O}(H) \otimes_{k} \bar{k}$. Thus, $\chi$ is defined over a smallest finite subextension $K / k$ of $\bar{k} / k$ : the field of definition, $K=k(\chi)$ (generated by the coordinates of $\chi$ in a $\bar{k}$-basis of $\mathcal{O}\left(H_{\bar{k}}\right)$ consisting of elements of $\mathcal{O}(H))$. So $\chi$ defines a morphism of $K$-group schemes $H_{K} \rightarrow \mathbb{G}_{m, K}$, or equivalently a morphism of $k$-group schemes

$$
\rho(\chi): H \longrightarrow \mathrm{R}_{K / k}\left(\mathbb{G}_{m, K}\right),
$$

where $\mathrm{R}_{K / k}$ denotes the Weil restriction of scalars: for any scheme $S$, the group of $S$-points $\mathrm{R}_{K / k}\left(\mathbb{G}_{m, K}\right)(S)$ is the unit group of the $K$-algebra $\mathcal{O}(S) \otimes_{k} K$ (see [CGP15, A.5] for generalities on Weil restriction). Thus, we may view $\rho(\chi)$ as a linear representation of $H$ in the $k$-vector space $K$, in which $\mathrm{R}_{K / k}\left(\mathbb{G}_{m, K}\right)$ acts by multiplication.

\section{Proposition 3.1. \\ (i) The representation $\rho(\chi)$ is irreducible, with commutant} algebra $k(\chi)$.

(ii) Every irreducible representation of $H$ is isomorphic to $\rho(\chi)$ for some character $\chi$ of $H$.

Proof. We argue as in the proof of [Wa79, 9.4]. Let $\rho: H \rightarrow \mathrm{GL}(V)$ be a finitedimensional representation. Consider the dual representation $\rho^{\vee}$ of $H$ in $V^{\vee}$, and the corresponding comodule map

$$
\Delta_{\rho} \vee: V^{\vee} \longrightarrow V^{\vee} \otimes_{k} \mathcal{O}(H) .
$$

Since $H$ is commutative, $\Delta_{\rho} \vee$ is equivariant for the $H$-representation on $V^{\vee}$, and that on $V^{\vee} \otimes_{k} \mathcal{O}(H)$ via its action on $V^{\vee}$. Also, the matrix coefficients of $\rho^{\vee}$ generate a subspace $C=C\left(\rho^{\vee}\right) \subset \mathcal{O}(H)$ which is stable under the comultiplication $\Delta$ : $\mathcal{O}(H) \rightarrow \mathcal{O}(H) \otimes_{k} \mathcal{O}(H)$; hence $C$ is a finite-dimensional sub-coalgebra. Note that $C$ is co-commutative and has a co-unit, since these properties hold for $\mathcal{O}(H)$. Thus, the dual vector space $C^{\vee}$ is a finite-dimensional commutative algebra with unit, and $V$ is a $C^{\vee}$-module. Moreover, $C^{\vee}$ acts on $V$ by $H$-invariant endomorphisms, since the transpose map $V^{\vee} \rightarrow V^{\vee} \otimes_{k} C$ is equivariant for the $H$-representations as above. Also, by [DG70, II.2.2.4], a subspace $W \subset V^{\vee}$ is $H$-stable if and only if $\Delta_{\rho} \vee(W) \subset W \otimes_{k} C$.

We now assume that $\rho$ is irreducible; then so is $\rho^{\vee}$, and hence $V^{\vee}$ is a simple $C$ comodule. Equivalently, $V$ is a simple $C^{\vee}$-module. This yields an isomorphism of $C^{\vee}$-modules $V \simeq K$ for some quotient field $K$ of $C^{\vee}$; moreover, $H$ acts linearly on $K$ and commutes with the $K$-action by multiplication. Thus, $\rho$ is identified with a 
morphism of $k$-group schemes $H \rightarrow \mathrm{R}_{K / k}\left(\mathbb{G}_{m, K}\right)$, which corresponds to a morphism of $K$-group schemes $H_{K} \rightarrow \mathbb{G}_{m, K}$, or equivalently to a character $\chi \in \mathrm{X}(H)$, defined over $K$. If $\chi$ is defined over a smaller subfield $L$ containing $k$, then the corresponding morphism of $L$-group schemes $H_{L} \rightarrow \mathbb{G}_{m, L}$ yields a factorization of $\rho$ through a morphism $H \rightarrow \mathrm{R}_{L / k}\left(\mathbb{G}_{m, L}\right)$. In particular, $H$ stabilizes the subspace $L$ of $K$. As $\rho$ is irreducible, it follows that $L=K$; equivalently, $K=k(\chi)$ and $\rho=\rho(\chi)$. This shows (ii).

To prove (i), we may assume that $\rho(\chi)$ is a monomorphism, and hence view $H$ as a $k$-subgroup scheme of $\mathrm{R}_{K / k}\left(\mathbb{G}_{m, K}\right)$, where $K=k(\chi)$. For the representation of $\mathrm{R}_{K / k}\left(\mathbb{G}_{m, K}\right)$ in $K$, the co-algebra of matrix coefficients is easily checked to be $K^{\vee}$ (the $k$-linear dual of $K$ ). As a consequence, the co-algebra $C$ of matrix coefficients of $\rho(\chi)$ is a quotient of $K^{\vee}$; equivalently, $C^{\vee}$ is a $k$-subalgebra of $K$, and hence a subfield, say $L$. Moreover, we have a commutative diagram of morphisms of $k$-algebras

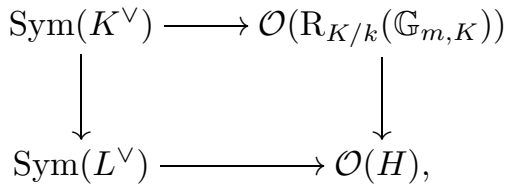

which translates into a commutative diagram of morphisms of $k$-schemes

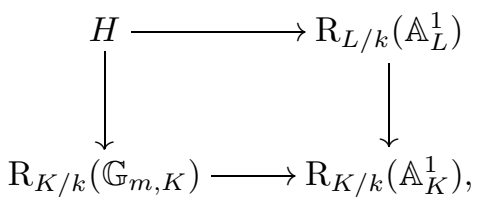

where the vertical arrows and the bottom horizontal arrow are immersions. Hence so is the top horizontal arrow. As a consequence, $H$ acts linearly on $K$ via a morphism to $R_{L / k}\left(\mathbb{G}_{m, L}\right)$, and $\chi$ is defined over $L$. Thus, $L=K=C^{\vee}$. Using again the characterization of the $H$-stable subspaces of $V^{\vee}$ in terms of the comodule map, it follows that $V$ is irreducible. In particular, its commutant algebra is a division algebra $D$ of finite dimension over $k$, say $d$. We have $d \geq[K: k]$ as $D \supset K$, and $d \leq[K: k]$ as $K$ is a $D$-module. So equality holds, and $D=K$.

Next, consider the absolute Galois group

$$
\Gamma:=\operatorname{Gal}\left(k_{s} / k\right)=\operatorname{Aut}(\bar{k} / k),
$$

and its continuous action on the character group $\mathrm{X}(H)$; denote this action by $(\gamma, \chi) \mapsto \gamma \cdot \chi$. Note that $k(\gamma \cdot \chi)=\gamma k(\chi)$ for any $\gamma \in \Gamma$ and $\chi \in \mathrm{X}(H)$.

Also, recall that $H$ lies in a unique exact sequence of commutative affine group schemes

$$
0 \longrightarrow M \longrightarrow H \longrightarrow U \longrightarrow 0
$$

where $M$ is of multiplicative type and $U$ is unipotent; the formation of $M, U$ commutes with base change under field extensions. For any such extension $k^{\prime} / k$ with $k^{\prime}$ perfect, the induced exact sequence

$$
0 \longrightarrow M_{k^{\prime}} \longrightarrow H_{k^{\prime}} \longrightarrow U_{k^{\prime}} \longrightarrow 0
$$


has a unique splitting; in particular, (3.2) splits over $\bar{k}$. Since every character of $U$ is trivial, this yields a $\Gamma$-equivariant isomorphism

$$
\mathrm{X}(H) \longrightarrow \mathrm{X}(M),\left.\quad \chi \longmapsto \chi\right|_{M}
$$

where the $\Gamma$-group $\mathrm{X}(M)$ may be identified with the Cartier dual $\mathrm{D}(M)$. Finally, recall from [DG70, II.3.3.7, II.3.4.3] that every representation of $M$ is completely reducible.

Lemma 3.2. Let $\chi \in \mathrm{X}(H)$ and denote by $\Gamma_{\chi}$ its stabilizer in $\Gamma$. Set $K:=k(\chi)$, $\eta:=\left.\chi\right|_{M}$, and $L:=k(\eta)$.

(i) $L=K \cap k_{s}$.

(ii) $L$ is the fixed subfield of $\Gamma_{\chi}$ in $k_{s}$.

(iii) $\operatorname{res}_{M}^{H} \rho(\chi) \simeq[K: L] \rho(\eta)$.

Proof. (i) If $p=0$, then the assertion follows readily from the isomorphism $H \simeq$ $M \times U$. Thus, we may assume $p>0$.

Clearly, $\eta$ is defined over $K$; that is, $L \subset K$. Also, as $M$ is of multiplicative type, its characters are all defined over $k_{s}$; in particular, $L$ is separable over $k$. To complete the proof, it suffices to show that $K^{p^{n}} \subset L$ for $n \gg 0$. For this, we may replace $k$ with $L$, and hence assume that $\eta \in \operatorname{Hom}_{\widetilde{\mathcal{L}}}\left(M, \mathbb{G}_{m}\right) . \operatorname{As~}_{\operatorname{Hom}_{\widetilde{\mathcal{L}}}}\left(U, \mathbb{G}_{m}\right)=0$, we have an exact sequence

$$
0 \longrightarrow \operatorname{Hom}_{\tilde{\mathcal{L}}}\left(H, \mathbb{G}_{m}\right) \longrightarrow \operatorname{Hom}_{\tilde{\mathcal{L}}}\left(M, \mathbb{G}_{m}\right) \longrightarrow \operatorname{Ext}_{\widetilde{\mathcal{L}}}^{1}\left(U, \mathbb{G}_{m}\right)
$$

Also, $U$ is the filtered inverse limit of its algebraic quotients $U^{\prime}$. Together with [DG70, V.2.3.9], this yields

$$
\operatorname{Ext}_{\widetilde{\mathcal{L}}}^{1}\left(U, \mathbb{G}_{m}\right) \simeq \lim _{\rightarrow} \operatorname{Ext}_{\widetilde{\mathcal{L}}}^{1}\left(U^{\prime}, \mathbb{G}_{m}\right) .
$$

Since each $U^{\prime}$ is a unipotent algebraic group, $\operatorname{Ext}_{\widetilde{\mathcal{L}}}^{1}\left(U^{\prime}, \mathbb{G}_{m}\right)$ is killed by a power of $p$. Thus, so is every element of $\operatorname{Ext}_{\widetilde{\mathcal{L}}}^{1}\left(U, \mathbb{G}_{m}\right)$; hence $p^{n} \eta$ extends to a morphism $H \rightarrow \mathbb{G}_{m}$ for $n \gg 0$. This implies the assertion.

(ii) We view $\eta$ as an element of $\mathcal{O}\left(M_{k_{s}}\right)=\mathcal{O}(M) \otimes_{k} k_{s}$. Write accordingly $\eta=\sum_{i=1}^{n} f_{i} \otimes z_{i}$, where $f_{1}, \ldots, f_{n} \in \mathcal{O}(M)$ are linearly independent over $k$ and $z_{1}, \ldots, z_{n} \in k_{s}$. Then $k(\eta)=k\left(z_{1}, \ldots, z_{n}\right)$. Also, the stabilizer in $\Gamma$ of $\chi$ equals that of $\eta$, and hence consists of the $\gamma$ such that $\gamma z_{i}=z_{i}$ for $i=1, \ldots, n$. Thus, $\gamma \in \Gamma_{\chi}$ if and only if $\gamma$ fixes $L$ pointwise. So the assertion follows from Galois theory.

(iii) This is a direct consequence of the definition of $\rho(\chi)$.

Proposition 3.3. Let $\chi, \chi^{\prime} \in \mathrm{X}(H)$. Then we have $\rho\left(\chi^{\prime}\right) \simeq \rho(\chi)$ if and only if $\chi^{\prime} \in \Gamma \cdot \chi$.

Proof. Assume that $\chi^{\prime}=\gamma \cdot \chi$ for some $\gamma \in \Gamma$. We may choose a finite subextension $K^{\prime} / k$ which is stable under $\Gamma$ and contains $k(\chi)$. Then $\Gamma$ acts by automorphisms on the $k$-group scheme $\mathrm{R}_{K^{\prime} / k}\left(\mathbb{G}_{m, K^{\prime}}\right)$, and $\gamma$ restricts to an isomorphism

$$
\mathrm{R}_{k(\chi) / k}\left(\mathbb{G}_{m, k(\chi)}\right) \stackrel{\simeq}{\longrightarrow} \mathrm{R}_{k\left(\chi^{\prime}\right) / k}\left(\mathbb{G}_{m, k\left(\chi^{\prime}\right)}\right)
$$

which intertwines $\rho(\chi)$ and $\rho\left(\chi^{\prime}\right)$.

Conversely, assume that $\rho\left(\chi^{\prime}\right)$ is isomorphic to $\rho(\chi)$. Then the commutant algebras are isomorphic as well, hence $k\left(\chi^{\prime}\right) \simeq k(\chi)$ as extensions of $k$ (Proposition 3.1). In view of Lemma 3.2, it follows that $\rho\left(\left.\chi^{\prime}\right|_{M}\right) \simeq \rho\left(\left.\chi\right|_{M}\right)$. Consider the diagonalizable $k_{s}$-group scheme $M_{k_{s}}$; then its representation $\rho\left(\left.\chi\right|_{M}\right)_{k_{s}}$ decomposes into the direct sum of one-dimensional representations with weights the $\left.\gamma \cdot \chi\right|_{M}$, where 
$\gamma \in \Gamma$ (since the algebra $k\left(\left.\chi\right|_{M}\right) \otimes_{k} k_{s}$ decomposes into the direct sum of twists of $k_{s}$ by elements of $\left.\Gamma\right)$. Using the analogous decomposition for $\rho\left(\left.\chi^{\prime}\right|_{M}\right)_{k_{s}}$, it follows that $\left.\Gamma \cdot \chi^{\prime}\right|_{M}=\left.\Gamma \cdot \chi\right|_{M}$. Hence $\chi^{\prime} \in \Gamma \cdot \chi$.

3.2. Blocks. In this subsection, we study the decomposition of the category $H$-mod into blocks; the main result (Proposition [3.9) describes the structure of each block in terms of twisted representations of the largest unipotent quotient $U$. We start with a key observation.

Proposition 3.4. Let $\chi, \chi^{\prime} \in \mathrm{X}(H)$. If $\chi^{\prime} \notin \Gamma \cdot \chi$, then $\operatorname{Ext}_{H}^{1}\left(k(\chi), k\left(\chi^{\prime}\right)\right)=0$.

Proof. By Lemma 3.2 we have $\operatorname{res}_{M}^{H} k(\chi) \simeq n k\left(\left.\chi\right|_{M}\right), \operatorname{res}_{M}^{H} k\left(\chi^{\prime}\right) \simeq n^{\prime} k\left(\left.\chi^{\prime}\right|_{M}\right)$ for positive integers $n, n^{\prime}$; moreover, the simple $M$-modules $k\left(\left.\chi\right|_{M}\right), k\left(\left.\chi^{\prime}\right|_{M}\right)$ are non-isomorphic. Thus, every extension of $H$-modules

$$
0 \longrightarrow k\left(\chi^{\prime}\right) \longrightarrow E \longrightarrow k(\chi) \longrightarrow 0
$$

has a unique splitting as an extension of $M$-modules; in particular, the space $\operatorname{Hom}_{M}(k(\chi), E)$ is non-zero. As this space is a module under the unipotent group scheme $U=H / M$, it contains a non-zero $U$-invariant; hence $\operatorname{Hom}_{H}(k(\chi), E) \neq 0$. As a consequence, the above extension splits.

Combining Proposition 3.4 with [Ja03, II.7.1], we obtain a decomposition of the category of finite-dimensional $H$-modules into blocks,

$$
H-\bmod =\bigoplus_{\chi \in \mathrm{X}(H) / \Gamma} H-\bmod _{\chi},
$$

where $H-\bmod _{\chi}$ denotes the full subcategory of $H$-mod consisting of the modules having all their composition factors isomorphic to $k(\chi)$. Each $H-\bmod _{\chi}$ is an abelian category; it has a unique simple object, $k(\chi)$, which is its own commutant algebra (Proposition 3.1).

Taking for $\chi$ the trivial character, we obtain the principal block $H$-mod ${ }_{0}$, consisting of the modules with trivial composition factors. By Lemma 3.2, these are exactly the modules fixed pointwise by $M$, or equivalently the modules under $H / M=U$. This yields an equivalence

$$
H-\bmod _{0} \simeq U-\bmod ,
$$

where the right-hand side is the category of unipotent representations.

Remark 3.5. Proposition 3.4 together with the block decomposition (3.3) easily imply that $\operatorname{Ext}_{H}^{i}\left(k(\chi), k\left(\chi^{\prime}\right)\right)=0$ for all $i \geq 1$ and $\chi, \chi^{\prime} \in \mathrm{X}(H)$ such that $\chi^{\prime} \notin \Gamma \cdot \chi$. Also, we have canonical isomorphisms

$$
\operatorname{Ext}_{H}^{i}(k(\chi), k(\chi)) \simeq \mathrm{H}^{i}\left(H, k(\chi)^{\vee} \otimes k(\chi)\right) \simeq \mathrm{H}^{i}\left(U, \operatorname{End}_{M} k(\chi)\right),
$$

where the first equality holds by [Ja03, I.4.4], and the second one follows from the Hochschild-Serre spectral sequence (see [Ja03, I.6.6]) in view of the complete reducibility of representations of $M$.

If $k(\chi)$ is separable over $k$, then $k(\chi)$ is irreducible as an $M$-module, and hence $\operatorname{End}_{M} k(\chi)=k(\chi)$ by Proposition 3.1. This yields canonical isomorphisms

$$
\operatorname{Ext}_{H}^{i}(k(\chi), k(\chi)) \cong \mathrm{H}^{i}(U, k) \otimes_{k} k(\chi), \quad(i \geq 0),
$$

which also follow from Theorem 3.11 below. As $\mathrm{H}^{1}(U, k) \neq 0$ for any non-trivial unipotent group $U$ (see, e.g., DG70, II.3.7, IV.2.5]), it follows that the category $H-\bmod _{\chi}$ is semisimple if and only if $U=0$. 
For an arbitrary character $\chi$, it would be interesting to find an explicit description of $\operatorname{Ext}_{H}^{1}(k(\chi), k(\chi))$, and to deduce an effective criterion for its vanishing (which is equivalent to the category $H-\bmod _{\chi}$ being semisimple). We will obtain a characterization of the semisimplicity of $H-\bmod _{\chi}$ by an alternative approach, in Lemma 3.7

The block decomposition (3.3) extends to a decomposition of the category of $H$-modules,

$$
H-\operatorname{Mod}=\bigoplus_{\chi \in \mathrm{X}(H) / \Gamma} H-\operatorname{Mod}_{\chi}
$$

where $H-\operatorname{Mod}_{\chi}$ consists of the direct limits of objects of $H-\bmod _{\chi}$. In particular, we obtain a decomposition of the regular representation,

$$
\mathcal{O}(H)=\bigoplus_{\chi \in \mathrm{X}(H) / \Gamma} \mathcal{O}(H)_{\chi}
$$

Lemma 3.6. (i) For any $H$-module $V$, we have a natural isomorphism of $H$ modules

$$
\bigoplus_{\chi \in \mathrm{X}(H) / \Gamma} \operatorname{Hom}_{M}\left(k\left(\left.\chi\right|_{M}\right), V\right) \stackrel{\simeq}{\longrightarrow} V, \quad f \longmapsto f(1),
$$

where $H$ acts on $\operatorname{Hom}_{M}\left(k\left(\left.\chi\right|_{M}\right), V\right)$ via its action on $V$.

(ii) Each $\operatorname{Hom}_{M}\left(k\left(\left.\chi\right|_{M}\right), V\right)$ is an object of $H-\operatorname{Mod}_{\chi}$.

(iii) Each $\mathcal{O}(H)_{\chi}$ is the injective hull of $k(\chi)$ in $H-\operatorname{Mod}_{\chi}$.

Proof. (i) As the $M$-module $\operatorname{res}_{M}^{H} V$ is semisimple and the simple $M$-modules are exactly the $k\left(\left.\chi\right|_{M}\right)$, where $\chi$ is uniquely determined up to the $\Gamma$-action, we have an isomorphism

$$
\bigoplus_{\chi \in \mathrm{X}(H) / \Gamma} \operatorname{Hom}_{M}\left(k\left(\left.\chi\right|_{M}\right), V\right) \otimes_{\operatorname{End}_{M}\left(k\left(\left.\chi\right|_{M}\right)\right)} k\left(\left.\chi\right|_{M}\right) \stackrel{\simeq}{\longrightarrow} V,
$$

which takes every $f \otimes v$ to $f(v)$. Moreover, $\operatorname{End}_{M}\left(k\left(\left.\chi\right|_{M}\right)\right)$ is identified with $k\left(\left.\chi\right|_{M}\right)$ acting by multiplication, in view of Proposition 3.1 This yields the assertion.

(ii) By construction, $\operatorname{res}_{M}^{H} \operatorname{Hom}_{M}\left(k\left(\left.\chi\right|_{M}\right), V\right)$ is a direct sum of copies of $k\left(\left.\chi\right|_{M}\right)$. This implies the statement in view of Lemma 3.2(iii).

(iii) Since $\mathcal{O}(H)$ is an injective object of $H$-Mod, we see that $\mathcal{O}(H)_{\chi}$ is an injective object of $H-\operatorname{Mod}_{\chi}$. So it suffices to show that $\mathcal{O}(H)_{\chi}$ contains a unique copy of $k(\chi)$. But

$$
\operatorname{Hom}_{H}\left(k(\chi), \mathcal{O}(H)_{\chi}\right)=\operatorname{Hom}_{H}(k(\chi), \mathcal{O}(H)) \simeq k(\chi)^{\vee},
$$

where the isomorphism holds by Frobenius reciprocity (see [Ja03, I.3.7]). Thus, $\operatorname{Hom}_{H}\left(k(\chi), \mathcal{O}(H)_{\chi}\right)$ is a $k$-vector space of dimension $[k(\chi): k]$, and hence a vector space of dimension 1 under $\operatorname{End}_{H} k(\chi)=k(\chi)$. This yields the desired assertion.

Next, choose $\chi \in \mathrm{X}(H)$ and use the notation $K, \eta, L$ of Lemma 3.2. Recall from that lemma that $L$ is the separable closure of $k$ in $K$; in particular, the degree $[K: L]$ is a power of the characteristic exponent of $k$. We view $K$ as a simple $H$-module via $\rho(\chi)$; likewise, $L$ is a simple $M$-module via $\rho(\eta)$. 
Lemma 3.7. $\quad$ (i) $\mathcal{O}(H)_{\chi} \simeq \operatorname{ind}_{M}^{H}(L)$ as $H$-modules.

(ii) The category $H-\bmod _{\chi}$ is semisimple if and only if the group scheme $U$ is finite of order $[K: L]$.

Proof. (i) We argue as in the proof of Lemma 3.6(iii). By Frobenius reciprocity (see [Ja03, I.3.4]), we have

$$
\operatorname{Hom}_{H}\left(V, \operatorname{ind}_{M}^{H} L\right) \simeq \operatorname{Hom}_{M}\left(\operatorname{res}_{M}^{H} V, L\right)
$$

for any $H$-module $V$. Since every $M$-module is semisimple, it follows that $\operatorname{ind}_{M}^{H} L$ is injective in $H$-Mod. Also, if $V$ is simple and not isomorphic to $K$, then we have $\operatorname{Hom}_{M}\left(\operatorname{res}_{M}^{H} L, V\right)=0$ by Lemma $3.2\left(\right.$ iii). Thus, $\operatorname{ind}_{M}^{H} L$ is an object of $H-\operatorname{Mod}_{\chi}$. Finally,

$$
\operatorname{Hom}_{H}\left(K, \operatorname{ind}_{M}^{H} L\right) \simeq \operatorname{Hom}_{M}(K, L)=\operatorname{Hom}_{L}(K, L),
$$

where the equality follows from the fact that $\operatorname{End}_{M}(L)=L$ (Proposition 3.1). Thus, $\operatorname{Hom}_{H}\left(K, \operatorname{ind}_{M}^{H}(L)\right)$ is an $L$-vector space of dimension $[K: L]$, and hence a $k$-vector space of dimension $[K: k]$. Since the $k$-vector space $\operatorname{End}_{H}(K)$ has dimension $[K: k]$ as well (Proposition 3.1 again), this yields the desired assertion in view of [Ja03, I.3.18].

(ii) The semisimplicity of $H-\bmod _{\chi}$ is equivalent to that of $H-M_{\chi} d_{\chi}$, and the latter holds if and only if the injective cogenerator $\mathcal{O}(H)_{\chi}$ is semisimple; by Lemma 3.6(iii), this is equivalent to the equality $\operatorname{dim} \mathcal{O}(H)_{\chi}=[K: k]$. Also,

$$
\operatorname{dim} \mathcal{O}(H)_{\chi}=\operatorname{dim}\left(\mathcal{O}(H) \otimes_{k} L\right)^{M}=\operatorname{dim}_{\bar{k}}\left(\mathcal{O}\left(H_{\bar{k}}\right) \otimes_{\bar{k}} L_{\bar{k}}\right)^{M_{\bar{k}}}=\operatorname{dim}_{\bar{k}} \mathcal{O}\left(U_{\bar{k}}\right) \otimes_{\bar{k}} L_{\bar{k}},
$$

where the latter equality follows from the isomorphism $H_{\bar{k}} \simeq U_{\bar{k}} \times M_{\bar{k}}$. Thus, $\operatorname{dim} \mathcal{O}(H)_{\chi}=[L: k] \operatorname{dim} \mathcal{O}(U)$; this implies the assertion.

Example 3.8. Assume that $k$ is imperfect and separably closed. We may then choose $t \in k \backslash k^{p}$. Let $V \subset \mathbb{G}_{a} \times \mathbb{G}_{a}$ be the zero subscheme of $y^{p}-x-t x^{p}$. Then $V$ is a non-trivial $k$-form of $\mathbb{G}_{a}$; in view of [To13, Lem. 9.4], it follows that there exists a non-trivial extension

$$
\xi: \quad 0 \longrightarrow \mathbb{G}_{m} \longrightarrow E \longrightarrow V \longrightarrow 0 .
$$

On the other hand, the projection $x: V \rightarrow \mathbb{G}_{a}$ lies in an exact sequence

$$
0 \longrightarrow \alpha_{p} \longrightarrow V \longrightarrow \mathbb{G}_{a} \longrightarrow 0,
$$

where $\alpha_{p}$ denotes the kernel of the Frobenius endomorphism of $\mathbb{G}_{a}$. The pullback of $\xi$ by $\alpha_{p} \rightarrow V$ yields an extension

$$
0 \longrightarrow \mathbb{G}_{m} \longrightarrow H \longrightarrow \alpha_{p} \longrightarrow 0,
$$

which is non-trivial as well; indeed, the pullback map

$$
\operatorname{Ext}_{\mathcal{C}}^{1}\left(V, \mathbb{G}_{m}\right) \longrightarrow \operatorname{Ext}_{\mathcal{C}}^{1}\left(\alpha_{p}, \mathbb{G}_{m}\right)
$$

is injective in view of the vanishing of $\operatorname{Ext}_{\mathcal{C}}^{1}\left(\mathbb{G}_{a}, \mathbb{G}_{m}\right)$ (which follows, e.g., from [DG70, III.6.2.5, III. 6.5.1]). Thus, the canonical character of $\mathbb{G}_{m}$ extends uniquely to a character $\chi$ of $H$, which is not defined over $k$. But $\chi$ is defined over the subfield $K:=k\left(t^{1 / p}\right)$, since $V_{K} \simeq\left(\mathbb{G}_{a}\right)_{K}$ and hence $\xi$ splits over $K$. As $[K: k]=p$, it follows that $k(\chi)=K$. Since $k\left(\left.\chi\right|_{\mathbb{G}_{m}}\right)=k$ and $\alpha_{p}$ has order $p$, Lemma 3.7(ii) yields that the category $H-\bmod _{\chi}$ is semisimple. 
The exact sequence (3.2) yields an exact sequence of $L$-group schemes

$$
0 \longrightarrow M_{L} \longrightarrow H_{L} \longrightarrow U_{L} \longrightarrow 0 \text {. }
$$

Viewing $\eta$ as a morphism $M_{L} \rightarrow \mathbb{G}_{m, L}$, this yields in turn a pushout diagram of such group schemes

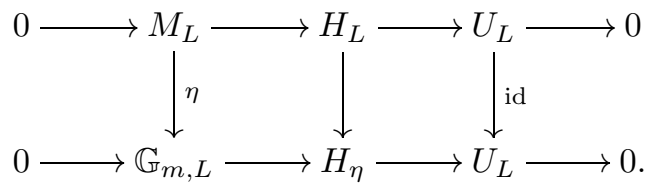

Also, every object $V$ of $H-\operatorname{Mod}_{\chi}$ may be identified with $\operatorname{Hom}_{M}(L, V)$ in view of Lemma 3.6. Thus, $V$ has the structure of an $L$-vector space equipped with a compatible action of $H$, i.e., of an $H_{L}$-module. Since $M_{L}$ acts on $V$ via $\eta$, the representation of $H_{L}$ in $V$ factors uniquely through a representation of the pushout $H_{\eta}$. Denote by $F(V)$ the corresponding $H_{\eta}$-module, and by $H_{\eta}$ - $\bmod _{1}$ the block of $H_{\eta}$-mod associated with the identity character of $\mathbb{G}_{m, L}$. We may view this block as that of twisted $U_{L}$-modules, where the twist is defined by the extension in the bottom line of (3.5).

Proposition 3.9. The assignment $V \mapsto F(V)$ extends to an exact functor, which yields an equivalence of categories

$$
F: H-\bmod _{\chi} \longrightarrow H_{\eta}-\bmod _{1} \text {. }
$$

Proof. Consider a morphism of $H$-modules $f: V \rightarrow W$. This defines a map

$$
F(f): \operatorname{Hom}_{M}(L, V) \longrightarrow \operatorname{Hom}_{M}(L, W), \quad u \longmapsto f \circ u .
$$

We claim that $F(f)$ is a morphism of $H_{\eta}$-modules. It suffices to check that $F(f)$ is $L$-linear, where $L$ acts on $\operatorname{Hom}_{M}(L, V), \operatorname{Hom}_{M}(L, W)$ via multiplication on itself. But this follows by identifying the $L$-modules $V, W$ with direct sums of copies of $L$, and using the fact that $\operatorname{End}_{M}(L)=L$ (Proposition 3.1).

The claim defines the functor $F$ on morphisms; one may readily check that $F$ is exact, fully faithful, and essentially surjective.

Next, we introduce a class of characters for which the associated block has an especially simple structure. We say that $\chi \in \mathrm{X}(H)$ is separable if its field of definition is a separable extension of $k$. Also, recall that an $H$-module $V$ is said to be absolutely semisimple if the $H_{\bar{k}}$-module $V_{\bar{k}}:=V \otimes_{k} \bar{k}$ is semisimple.

Lemma 3.10. With the notation of Lemma 3.2, the following conditions are equivalent for a character $\chi$ :

(i) $\chi$ is separable.

(ii) $L=K$.

(iii) The representation $\operatorname{res}_{M}^{H} \rho(\chi)$ is irreducible.

(iv) The extension $0 \rightarrow \mathbb{G}_{m, L} \rightarrow H_{\eta} \rightarrow U_{L} \rightarrow 0$ splits.

(v) The $H$-module $K$ is absolutely semisimple.

Proof. The equivalences (i) $\Leftrightarrow$ (ii) $\Leftrightarrow$ (iii) are obvious. Also, in view of the commutative diagram (3.5), the splittings of the extension in (iv) may be identified with the morphisms $H_{L} \rightarrow \mathbb{G}_{m, L}$ extending the identity character of $\mathbb{G}_{m, L}$, i.e., with the morphisms $H \rightarrow \mathrm{R}_{L / k}\left(\mathbb{G}_{m, L}\right)$ extending $\rho(\eta): M \rightarrow \mathrm{R}_{L / k}\left(\mathbb{G}_{m, L}\right)$; this implies the equivalence (ii) $\Leftrightarrow$ (iv). 
We now show the equivalence $(\mathrm{i}) \Leftrightarrow(\mathrm{v})$. If $\chi$ is separable, then the $\bar{k}$-algebra $K \otimes_{k} \bar{k}$ is isomorphic to a product of copies of $\bar{k}$. This yields a decomposition of the corresponding $H_{\bar{k}}$-module into $H_{\bar{k}}$-stable lines; in particular, this module is semisimple.

Conversely, if the $H$-module $K$ is absolutely semisimple, then so is its commutant algebra. By Proposition 3.1, it follows that the field extension $K / k$ is separable.

Theorem 3.11. Let $\chi$ be a separable character of $H$, with field of definition $K$. Then the abelian category $H-\bmod _{\chi}$ is equivalent to $U_{K}$-mod.

Proof. We may replace $k$ with $K=L$ and $H$ with $H_{\eta}$ in view of Proposition 3.9. Then Lemma 3.10 yields an isomorphism $H \simeq \mathbb{G}_{m} \times U$. Denote by $k_{1}$ the onedimensional $H$-module with weight 1 ; then the assignment $V \mapsto V \otimes k_{1}$ extends to the desired equivalence

$$
U-\bmod =H-\bmod _{0} \stackrel{\simeq}{\longrightarrow} H-\bmod _{1} .
$$

\section{ISOGENIES}

4.1. Functorial properties of universal affine covers. Consider a morphism of abelian varieties $\varphi: A \rightarrow B$.

Proposition 4.1. $\quad$ (i) There exist unique morphisms $G(\varphi): G_{A} \rightarrow G_{B}$ and $H(\varphi): H_{A} \rightarrow H_{B}$ such that the diagram of extensions

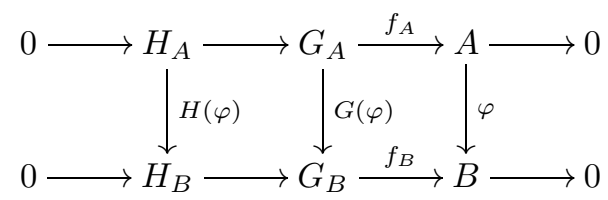

commutes. If $\varphi=n_{A}$ for some integer $n$, then $G(\varphi)=n_{G_{A}}, H(\varphi)=n_{H_{A}}$. Also, the formations of $G(\varphi), H(\varphi)$ commute with base change under field extensions.

(ii) For any morphism of abelian varieties $\psi: B \rightarrow C$, we have

$$
G(\psi \circ \varphi)=G(\psi) \circ G(\varphi), \quad H(\psi \circ \varphi)=H(\psi) \circ H(\varphi) .
$$

(iii) If $\varphi$ is an isogeny, then $G(\varphi)$ is an isomorphism. Moreover, there is an exact sequence of commutative affine group schemes

$$
0 \longrightarrow H_{A} \stackrel{H(\varphi)}{\longrightarrow} H_{B} \longrightarrow N \longrightarrow 0,
$$

where $N:=\operatorname{Ker}(\varphi)$.

Proof. (i) The existence of the morphisms $G(\varphi), H(\varphi)$ follows from the fact that $G_{A}$ is projective in the category $\widetilde{\mathcal{C}}$ of commutative quasi-compact group schemes. For the uniqueness, just note that every morphism $G_{A} \rightarrow H_{B}$ is zero, since $\mathcal{O}\left(G_{A}\right)=k$ and $H_{B}$ is affine. This uniqueness property implies the assertion on multiplication maps. Finally, the assertion on field extensions follows from Proposition 2.5.

(ii) This follows again from the uniqueness in (i).

(iii) There exists an isogeny $\psi: B \rightarrow A$ such that $\psi \circ \varphi$ is the multiplication map $n_{A}$ for some positive integer $n$, and $\varphi \circ \psi=n_{B}$. Then $G(\psi) \circ G(\varphi)=n_{G_{A}}$ by (ii); moreover, $n_{G_{A}}$ is an isomorphism in view of [Br18, Lem. 3.4]. Likewise, 
$G(\varphi) \circ G(\psi)=n_{G_{B}}$ is an isomorphism. This yields the assertion on $G(\varphi)$, and in turn the assertion on $H(\varphi)$ by applying the snake lemma to the commutative diagram (4.1).

Next, assume that $\varphi: A \rightarrow B$ is an isogeny and let $N:=\operatorname{Ker}(\varphi)$. Then the exact sequence (4.2) defines induction and restriction functors (see [Ja03, I.3]),

$$
\begin{aligned}
\text { ind } & =\operatorname{ind}_{H_{A}}^{H_{B}}: H_{A} \text {-mod } \longrightarrow H_{B} \text {-mod, } \\
\text { res } & =\operatorname{res}_{H_{A}}^{H_{B}}: H_{B} \text {-mod } \longrightarrow H_{A} \text {-mod. }
\end{aligned}
$$

Theorem 4.2. $\quad$ (i) For any homogeneous vector bundle $F$ on $B$, the pullback $\varphi^{*}(F)$ is a homogeneous vector bundle on $A$. Moreover, the assignment $F \mapsto \varphi^{*}(F)$ yields an exact functor $\varphi^{*}: \mathrm{HVec}_{B} \rightarrow \mathrm{HVec}_{A}$ which fits in a commutative square

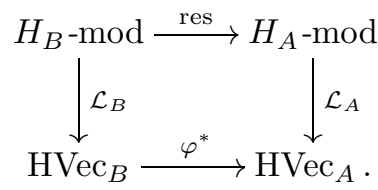

(ii) For any homogeneous vector bundle $E$ on $A$, the pushforward $\varphi_{*}(E)$ is a homogeneous vector bundle on $B$. Moreover, the assignment $E \mapsto \varphi_{*}(E)$ yields an exact functor $\varphi_{*}: \mathrm{HVec}_{A} \rightarrow \mathrm{HVec}_{B}$ which fits in a commutative square

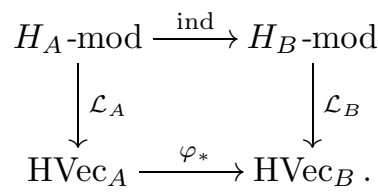

Proof. (i) Let $a \in A(\bar{k})$. Since $\varphi$ is a group homomorphism, we have $\tau_{a}^{*} \varphi^{*}(F) \simeq$ $\varphi^{*} \tau_{\varphi(a)}^{*}(F)$. As $F$ is homogeneous, it follows that $\tau_{a}^{*} \varphi^{*}(F) \simeq \varphi^{*}(F)$. So $\varphi^{*}(F)$ is homogeneous as well.

Clearly, the assignment $F \mapsto \varphi^{*}(F)$ extends to an exact functor $\operatorname{Vec}_{B} \rightarrow \operatorname{Vec}_{A}$, and hence to an exact functor $\varphi^{*}: \mathrm{HVec}_{B} \rightarrow \mathrm{HVec}_{A}$. The commutativity of the displayed square follows readily from the definitions.

(ii) Since $\varphi$ is finite and flat, it yields an exact functor $\varphi_{*}: \operatorname{Vec}_{A} \rightarrow \operatorname{Vec}_{B}$. Let $b \in B(\bar{k})$, and choose $a \in A(\bar{k})$ such that $b=\varphi(a)$; then the diagram

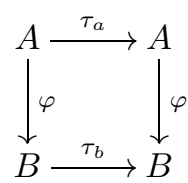

commutes, where the horizontal arrows are isomorphisms. It follows that $\tau_{b}^{*} \varphi_{*}(E) \simeq$ $\varphi_{*} \tau_{a}^{*}(E)$. Thus, $\varphi_{*}(E)$ is homogeneous.

By the projection formula, $\varphi_{*}$ is right adjoint to $\varphi^{*}$. Also, recall that ind is right adjoint to res (see [Ja03, I.3.4]). So the desired isomorphism of functors $\mathcal{L}_{B} \circ$ ind $\simeq \varphi_{*} \circ \mathcal{L}_{A}$ follows from the isomorphism $\mathcal{L}_{A} \circ$ res $\simeq \varphi^{*} \circ \mathcal{L}_{B}$ in view of the uniqueness of adjoints. 
Next, recall the dual exact sequence

$$
0 \longrightarrow \mathrm{D}(N) \longrightarrow \widehat{B} \stackrel{\widehat{\varphi}}{\longrightarrow} \widehat{A} \longrightarrow 0,
$$

where $\widehat{\varphi}$ denotes the dual isogeny, and $\mathrm{D}(N)$ the Cartier dual of the finite group scheme $N$. Also, recall the natural isomorphism $\operatorname{Hom}_{\widetilde{\mathcal{L}}}\left(H_{A_{k^{\prime}}}, \mathbb{G}_{m, k^{\prime}}\right) \simeq \widehat{A}\left(k^{\prime}\right)$ for any field extension $k^{\prime} / k$ (Lemma 2.6). Thus, we may identify the character group $\mathrm{X}\left(H_{A}\right)$ with $\widehat{A}(\bar{k})$, and the field of definition of any $x \in \mathrm{X}\left(H_{A}\right)$ with the residue field $k(x)$. In particular, the separable characters (as defined in 33.2 ) correspond to the points of $\widehat{A}\left(k_{s}\right)$. Also, the morphism $H(\varphi): H_{A} \rightarrow H_{B}$ defines a pullback map $H(\varphi)^{*}: \mathrm{X}\left(H_{B}\right) \rightarrow \mathrm{X}\left(H_{A}\right)$.

Lemma 4.3. For any $y \in \widehat{B}(\bar{k})$, we have $H(\varphi)^{*}(y)=\widehat{\varphi}(y)$ in $\widehat{A}(\bar{k})$.

Proof. Consider first the case where $y \in \widehat{B}(k)$. We then have a commutative diagram with exact rows

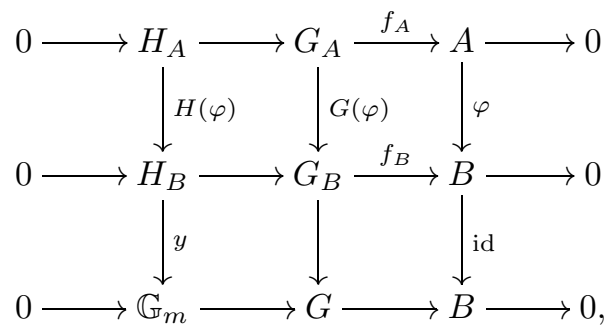

where the bottom line is obtained by pushout. This yields a commutative diagram

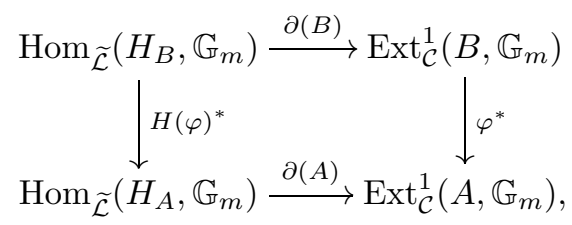

where the horizontal arrows are isomorphisms given by pushout. Also, the right vertical arrow may be identified with $\widehat{\varphi}: \widehat{B}(k) \rightarrow \widehat{A}(k)$. So we may rewrite the above square as

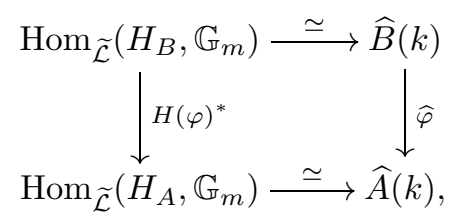

which yields the assertion in that case.

In the general case, we argue similarly by replacing $k$ with the residue field $k(y)$, and using compatibility with field extensions (Proposition 4.1).

Still considering $x \in \mathrm{X}\left(H_{A}\right)=\widehat{A}(\bar{k})$ with residue field $k(x)$, the representation of $H_{A}$ in $k(x)$ (constructed in 3.1) yields an associated homogeneous vector bundle $E(x):=\mathcal{L}_{A}(k(x))$ over $A$, of rank $[k(x): k]$. By Theorem 2.9 and Proposition 3.1 , $E(x)$ is irreducible; moreover, every irreducible homogeneous vector bundle on $A$ is obtained in this way. Also, given $x^{\prime} \in \widehat{A}(\bar{k})$, we have $E\left(x^{\prime}\right) \simeq E(x)$ if and only 
if $x^{\prime} \in \Gamma \cdot x$ (Proposition 3.3). Otherwise, $\operatorname{Ext}_{A}^{i}\left(E(x), E\left(x^{\prime}\right)\right)=0$ for any $i \geq 0$, by Theorem 2.12 and Proposition 3.4 .

If $x \in \widehat{A}(k)$, then $E(x)$ is just the corresponding (algebraically trivial) line bundle on $A$. For an arbitrary residue field $k(x)=: K$, we obtain a line bundle $L$ on $A_{K}$, and hence a vector bundle $\mathrm{R}_{K / k}(L)$ on $\mathrm{R}_{K / k}\left(A_{K}\right)$, of rank $[K: k]$; one may check that $E(x)$ is the pullback of that vector bundle under the canonical immersion $j_{A}: A \rightarrow \mathrm{R}_{K / k}\left(A_{K}\right)$ (see [CGP15, A.5.7]).

Likewise, we have irreducible homogeneous vector bundles $F(y)$ on $B$, indexed by the $\Gamma$-orbits in $\widehat{B}(\bar{k})$. We now determine their pullback to $A$.

Proposition 4.4. Let $y \in \widehat{B}(\bar{k})$ and set $x:=\widehat{\varphi}(y)$. Then we have $k(x) \subset k(y)$ and $\varphi^{*} F(y) \simeq[k(y): k(x)] E(x)$.

Proof. Clearly, $k(x) \subset k(y)$. Also, by Theorem 4.2, $\varphi^{*} F(y)$ is the homogeneous vector bundle associated with the representation of $H_{A}$ in $k(y)$ via the composition

$$
H_{A} \stackrel{H(\varphi)}{\longrightarrow} H_{B} \stackrel{\rho(y)}{\longrightarrow} \mathrm{R}_{k(y) / k}\left(\mathbb{G}_{m, k(y)}\right) .
$$

The associated character $\left(H_{A}\right)_{k(y)} \rightarrow \mathbb{G}_{m, k(y)}$ equals $x$ in view of Lemma 4.3. Thus, $H_{A}$ acts on $L$ via the corresponding morphism $H_{A} \rightarrow \mathrm{R}_{k(x) / k}\left(\mathbb{G}_{m, k(x)}\right)$. It follows that $\operatorname{res}_{H_{A}}^{H_{B}} \rho(y) \simeq[k(y): k(x)] \rho(x)$; this translates into the desired isomorphism.

The block decomposition (3.3) of $H_{A}$-mod yields a decomposition

$$
\operatorname{HVec}_{A}=\bigoplus_{x \in \widehat{A}(\bar{k}) / \Gamma} \operatorname{HVec}_{A, x}
$$

As a direct consequence of Proposition 4.4, we obtain the following.

Corollary 4.5. The pullback $\varphi^{*}: \mathrm{HVec}_{B} \rightarrow \mathrm{HVec}_{A}$ takes $\mathrm{HVec}_{B, y}$ to $\operatorname{HVec}_{A, \widehat{\varphi}(y)}$ for any $y \in \widehat{B}(\bar{k})$. Moreover, $\varphi^{*}$ preserves semisimplicity.

Next, we describe the pushforward of irreducible homogeneous bundles.

Proposition 4.6. (i) Let $x \in \widehat{A}(\bar{k})$ and consider the block decomposition

$$
\varphi_{*} E(x)=\bigoplus_{y \in \widehat{B}(\bar{k}) / \Gamma} F_{x, y} .
$$

Then $F_{x, y} \neq 0$ if and only if $\widehat{\varphi}(y) \in \Gamma \cdot x$. Under this assumption, $F_{x, y}$ contains a unique copy of $F(y)$; in particular, $F_{x, y}$ is indecomposable.

(ii) $\varphi_{*}$ preserves semisimplicity if and only if $\widehat{\varphi}$ is separable. Under this assumption, $F_{x, y}$ is irreducible for all $x, y$.

Proof. (i) Let $y \in \widehat{B}(\bar{k})$. Then

$$
\begin{gathered}
\operatorname{Hom}_{\mathrm{HVec}_{B}}\left(F(y), \varphi_{*} E(x)\right) \simeq \operatorname{Hom}_{\mathrm{HVec}_{A}}\left(\varphi^{*} F(y), E(x)\right) \\
\simeq[k(y): k(\widehat{\varphi}(y))] \operatorname{Hom}_{\mathrm{HVec}_{A}}(E(\widehat{\varphi}(y)), E(x)),
\end{gathered}
$$

where the first isomorphism holds by adjunction, and the second one follows from Proposition 4.4. Thus,

$$
\operatorname{dim}_{k} \operatorname{Hom}_{\mathrm{HVec}_{B}}\left(F(y), \varphi_{*} E(x)\right)=[k(y): k(\gamma \cdot x)][k(\gamma \cdot x): k]=[k(y): k]
$$


if $\widehat{\varphi}(y)=\gamma \cdot x$ for some $\gamma \in \Gamma$, and this dimension is zero otherwise. Since the $k$-vector space $\operatorname{Hom}_{\mathrm{HVec}_{B}}\left(F(y), \varphi_{*} E(x)\right)$ is a module under $\operatorname{End}_{\mathrm{HVec}_{B}} k(y) \simeq k(y)$, we see that $F_{x, y}$ contains a unique copy of $F(y)$ if $\widehat{\varphi}(y) \in \Gamma \cdot x$, and is zero otherwise.

(ii) Assume that $\varphi_{*}$ preserves semisimplicity; in particular, the homogeneous bundle $\varphi_{*}\left(\mathcal{O}_{A}\right)$ is semisimple. By Theorems 2.9 and 4.2 , it follows that the $H_{B^{-}}$ module $\mathcal{O}\left(H_{B} / H_{A}\right)=\mathcal{O}(N)$ is semisimple as well. Equivalently, $N$ is of multiplicative type (see [DG70, II.2.2.2, IV.3.3.6]), i.e., the Cartier dual $\mathrm{D}(N)$ is étale. In view of the exact sequence (4.3), this means that $\widehat{\varphi}$ is separable.

Conversely, assume that $\widehat{\varphi}$ is separable; then $N$ is of multiplicative type, as seen by reverting the above arguments. Note that $\varphi_{*}$ preserves semisimplicity if and only if so does $\operatorname{ind}_{H_{A}}^{H_{B}}$ (Theorem 4.2). We also need a general observation: let $H$ be an affine group scheme and let $V$ be an $H$-module; then $V$ is semisimple if and only if the $H_{k_{s}}$-module $V_{k_{s}}$ is semisimple. Indeed, $V$ is a module under the finitedimensional algebra $C^{\vee}$ constructed in the proof of Proposition 3.1 moreover, $V$ is semisimple as an $H$-module if and only if it is semisimple as a $C^{\vee}$-module (as follows from [DG70, II.2.2.4]). Since the formation of $C^{\vee}$ commutes with field extensions, this implies the observation by using the invariance of semisimplicity under separable extensions (see [Bo58, VIII.13.4]).

In view of this observation, we may assume that $k$ is separably closed. The fiber of $\widehat{\varphi}$ at any $x \in \widehat{A}(\bar{k})$ has $n$ distinct $\bar{k}$-points $y_{1}, \ldots, y_{n}$, where $n=\operatorname{deg}(\widehat{\varphi})=\operatorname{deg}(\varphi)$. Moreover, $k\left(y_{i}\right)=k(x)$ for $i=1, \ldots, n$ : indeed, $k\left(y_{i}\right)$ is a separable extension of $k(x)$ as $\widehat{\varphi}$ is étale, and $k\left(y_{i}\right) / k(x)$ is purely inseparable as $k=k_{s}$. By (i), $\varphi_{*} E(x)$ contains a sub-bundle isomorphic to $\bigoplus_{i=1}^{n} F\left(y_{i}\right)$. Since

$$
\operatorname{rk} \varphi_{*} E(x)=n[k(x): k]=\sum_{i=1}^{n}\left[k\left(y_{i}\right): k\right],
$$

it follows that $\varphi_{*} E(x) \simeq \bigoplus_{i=1}^{n} F\left(y_{i}\right)$. This completes the proof of preservation of semisimplicity under $\varphi_{*}$. The irreducibility of $F_{x, y}$ follows in view of (i).

Remark 4.7. To determine the pushforward of irreducible homogeneous vector bundles under an arbitrary isogeny $\varphi$, consider the exact sequence

$$
0 \longrightarrow M \longrightarrow N \longrightarrow U \longrightarrow 0,
$$

where $M$ is of multiplicative type and $U$ is unipotent. This yields a factorization $\varphi=\varphi_{u} \circ \varphi_{m}$, where $\varphi_{m}: A \rightarrow A / M$ has kernel $M$ and $\varphi_{u}: A / M \rightarrow B$ has kernel $U$, and a dual factorization $\widehat{\varphi}=\widehat{\varphi}_{m} \circ \widehat{\varphi}_{u}$, where $\widehat{\varphi}_{m}$ is separable and $\widehat{\varphi}_{u}$ is purely inseparable. As the pushforward $\left(\varphi_{m}\right)_{*} E(x)$ is described by Proposition 4.6. we may replace $\varphi$ with $\varphi_{u}$, and hence assume that $N=U$ is unipotent. Then $\widehat{\varphi}$ is bijective on $\bar{k}$-points; by Proposition 4.6 again, it follows that $\varphi_{*} E(x)$ is indecomposable for any $x \in \widehat{A}(\bar{k})$.

4.2. Unipotent vector bundles. Recall that a vector bundle $E$ over $A$ is unipotent if it admits a filtration $0=E_{0} \subset E_{1} \subset \cdots \subset E_{n}=E$, where each $E_{i}$ is a sub-bundle and $E_{i} / E_{i-1} \simeq \mathcal{O}_{A}$ for $i=1, \ldots, n$. The unipotent vector bundles form a full subcategory $\mathrm{UVec}_{A}$ of $\mathrm{Vec}_{A}$.

Theorem 4.8. (i) $\mathrm{UVec}_{A}=\mathrm{HVec}_{A, 0} \simeq U_{A}$-mod; in particular, $\mathrm{UVec}_{A}$ has a unique (up to isomorphism) simple object, $\mathcal{O}_{A}$. Moreover, we have isomorphisms of graded algebras

$$
\operatorname{Ext}_{\mathrm{UVec}}^{*}\left(\mathcal{O}_{A}, \mathcal{O}_{A}\right) \simeq \mathrm{H}^{*}\left(A, \mathcal{O}_{A}\right) \simeq \Lambda^{*} \mathrm{H}^{1}\left(A, \mathcal{O}_{A}\right) .
$$


(ii) $\mathrm{UVec}_{A}$ is an abelian tensor subcategory of $\mathrm{Vec}_{A}$, stable under extensions and direct summands.

(iii) We have an equivalence of abelian categories $\mathrm{HVec}_{A, x} \simeq \mathrm{UVec}_{A_{k(x)}}$ for any $x \in \widehat{A}\left(k_{s}\right)$.

Proof. (i) By Corollary 2.13, every unipotent vector bundle is homogeneous. This yieds the equality $\mathrm{UVec}_{A}=\mathrm{HVec}_{A, 0}$; the latter category is equivalent to $U_{A}$-mod by Theorem 2.9 and (3.4). This shows the first assertion. The second assertion is obtained by combining Theorem 2.12 and [Se97, Thm. VII.10].

(ii) This follows from (i) by using Corollary 2.10 ,

(iii) This is a direct consequence of Theorem 3.11

Remark 4.9. The unipotent vector bundles over an elliptic curve $A$ have been determined by Atiyah (when $k$ is algebraically closed), in the process of his description of all vector bundles over $A$; see [t57. In particular, there is a unique indecomposable unipotent bundle of rank $r$ for any integer $r \geq 0$. The decomposition of the tensor product of any two such bundles has been determined in At57] when $p=0$; the case where $p>0$ has been treated much more recently by Schröer (see [Sc10]).

Returning to an abelian variety $A$ of dimension $g$ over an arbitrary field $k$, recall that $U_{A} \simeq\left(\mathbb{G}_{a}\right)^{g}$ if $p=0$. As a consequence, the category $\mathrm{UVec}_{A}$ is equivalent to the category with objects the tuples $\left(r, X_{1}, \ldots, X_{g}\right)$, where $r$ is a non-negative integer and $X_{1}, \ldots, X_{g}$ are commuting nilpotent $r \times r$ matrices with coefficients in $k$; the morphisms from $\left(r, X_{1}, \ldots, X_{g}\right)$ to $\left(s, Y_{1}, \ldots, Y_{g}\right)$ are the $s \times r$ matrices $Z$ with coefficients in $k$ such that $Z X_{i}=Y_{i} Z$ for $i=1, \ldots, g$. An explicit description of the isomorphism classes of such tuples is well known for $g=1$, via the Jordan canonical form (which gives back the above results of Atiyah). But the higher-dimensional case is quite open; see [HH19] for a study of the moduli space of "regular" tuples.

This description of $\mathrm{UVec}_{A}$ in terms of linear algebra extends to an ordinary abelian variety $A$ over a separably closed field $k$ of characteristic $p>0$, since we then have $U_{A} \simeq\left(\mathbb{Z}_{p}\right)_{k}^{g}$ by (2.6).

Proposition 4.10. Let $\varphi: A \rightarrow B$ be an isogeny with kernel $N$.

(i) $\varphi^{*}: \mathrm{HVec}_{B} \rightarrow \mathrm{HVec}_{A}$ takes $\mathrm{UVec}_{B}$ to $\mathrm{UVec}_{A}$. If $\varphi$ is separable, then this yields an equivalence of categories $\mathrm{UVec}_{B} \simeq \mathrm{UVec}_{A}$.

(ii) $\varphi_{*}: \mathrm{HVec}_{A} \rightarrow \mathrm{HVec}_{B}$ takes $\mathrm{UVec}_{A}$ to $\bigoplus_{y \in \mathrm{X}(N) / \Gamma} \mathrm{HVec}_{B, y}$. In the resulting decomposition of $\varphi_{*}\left(\mathcal{O}_{A}\right)$, each summand is indecomposable.

Proof. (i) The first assertion holds since $\varphi^{*}$ is exact and takes $\mathcal{O}_{B}$ to $\mathcal{O}_{A}$. The second assertion follows from Theorem 4.2 in view of the exact sequence (4.2), which yields an isomorphism $U_{A} \stackrel{\simeq}{\longrightarrow} U_{B}$.

(ii) This follows similarly from the exactness of $\varphi_{*}$ and Proposition 4.6 .

Remark 4.11. Assume that $p>0$; then the $n$th relative Frobenius morphism $\mathrm{F}_{A}^{n}$ is a purely inseparable isogeny of degree $p^{n g}$. By Theorem 4.2 and Proposition 4.10, the decomposition of $\left(\mathrm{F}_{A}^{n}\right)_{*} \mathcal{O}_{A}$ into indecomposable summands corresponds to the block decomposition of $\mathcal{O}(N)$, where $N:=\operatorname{Ker}\left(\mathrm{F}_{A}^{n}\right)$ is an infinitesimal group scheme of order $p^{n g}$. Denoting by $r$ the $p$-rank of $A$, the largest subgroup scheme of multiplicative type of $N$ is a $k$-form of $\left(\mu_{p^{n}}\right)^{r}$ in view of (2.5). When (say) $k$ is separably closed, it follows that each block of $\mathcal{O}(N)$ has dimension $p^{n(g-r)}$; equivalently, $\left(\mathrm{F}_{A}^{n}\right)_{*} \mathcal{O}_{A}$ is the direct sum of $p^{n r}$ indecomposable summands of dimension $p^{n(g-r)}$. This gives back a recent result of Sannai and Tanaka (see [ST16, Thm. 1.2]). 
The analogous decomposition of $\left(\mathrm{F}_{A}^{n}\right)_{*}(L)$, obtained in [ST16, Thm. 5.3] for any $L \in \widehat{A}(k)$, can also be derived from Proposition 4.6 and Remark 4.7.

Still assuming $p>0$, and denoting by $\mathrm{V}_{A}^{n}: A^{\left(p^{n}\right)} \rightarrow A$ the $n$th Verschiebung, we obtain a characterization of unipotent vector bundles which refines a result of Miyanishi (see [Mi73, Rem. 2.4]).

Proposition 4.12. Let $E$ be a vector bundle over $A$. Then $E$ is unipotent if and only if $\left(\mathrm{V}_{A}^{n}\right)^{*} E$ is trivial for $n \gg 0$.

Proof. Assume that $E$ is unipotent. Then there exists a finite-dimensional representation $\rho: H_{A} \rightarrow \operatorname{GL}(V)$ such that $E=\mathcal{L}_{A}(V)$ and $\operatorname{Ker}(\rho) \supset M_{A}$. Thus, $H_{A} / \operatorname{Ker}(\rho)$ is a commutative unipotent algebraic group, and hence there exists $n_{0}$ such that $\mathrm{V}_{H_{A} / \operatorname{Ker}(\rho)}^{n}=0$ for $n \geq n_{0}$ (see [DG70, IV.3.4.11]). As a consequence, $\rho \circ \mathrm{V}_{H_{A}}^{n}$ is trivial for $n \geq n_{0}$. In view of the commutative diagram with exact rows

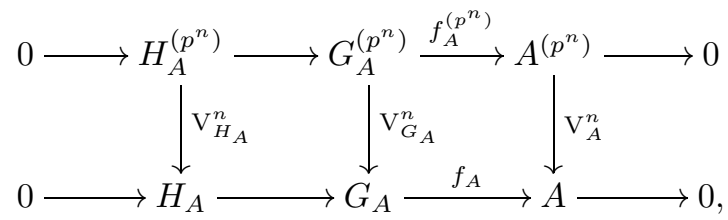

it follows that $\left(\mathrm{V}_{A}^{n}\right)^{*} E$ is trivial for $n \geq n_{0}$.

Conversely, assume that $\left(\mathrm{V}_{A}^{n}\right)^{*} E$ is trivial for $n \geq n_{0}$. By Theorem 4.2(i), it follows that $E \simeq G_{A} \times{ }^{H_{A}} V$, where $V$ is a finite-dimensional $H_{A}$-module which restricts trivially to $H_{A}^{\left(p^{n}\right)}$ via $H\left(\mathrm{~V}_{A}^{n}\right)$. Thus,

$$
E \simeq G_{A} / H_{A}^{\left(p^{n}\right)} \times{ }^{H_{A} / H_{A}^{\left(p^{n}\right)}} V \simeq A^{\left(p^{n}\right)} \times{ }^{\operatorname{Ker}\left(\mathrm{V}_{A}^{n}\right)} V .
$$

Since $\operatorname{Ker}\left(\mathrm{V}_{A}^{n}\right)$ is unipotent, it follows that $E$ is unipotent as well.

Remark 4.13. The essentially finite vector bundles $E$ over $A$, i.e., those such that $f^{*}(E)$ is trivial for some torsor $f: X \rightarrow A$ under a finite group scheme, admit a similar characterization. Indeed, as shown by Nori in No83, for any such bundle $E$, there exists $n>1$ such that $n_{a}^{*}(E)$ is trivial. As a consequence, the essentially finite vector bundles are exactly the iterated extensions of irreducible homogeneous vector bundles associated with torsion points of $\widehat{A}(\bar{k})$.

Also, $U_{A}$ is canonically isomorphic to the "nilpotent fundamental group scheme" $U(A, 0)$ introduced and studied by Nori in [No82, Chap. IV] in the more general setting of pointed schemes of finite type. This follows from [No82, Prop. 1]; note that the "nilpotent group schemes" considered there are exactly the unipotent group schemes in the sense of [DG70, IV.2.2].

\section{ACKNOWLEDGMENTS}

Many thanks to Corrado De Concini, Pedro Luis del Ángel Rodríguez, Gaël Rémond, Álvaro Rittatore, Catharina Stroppel, Tamás Szamuely, and Angelo Vistoli for very helpful discussions. Also, thanks to the anonymous referee for a careful reading and valuable comments. 


\section{REFERENCES}

[At57] M. F. Atiyah, Vector bundles over an elliptic curve, Proc. London Math. Soc. (3) 7 (1957), 414-452, DOI 10.1112/plms/s3-7.1.414. MR0131423

[Ay19] J. Ayoub, Topologie feuilletée et théorie de Galois différentielle, preprint, 2019.

[Bo58] N. Bourbaki, Éléments de mathématique, Fasc. XXIII, Hermann, Paris, 1973. Livre II: Algèbre. Chapitre 8: Modules et anneaux semi-simples; Nouveau tirage de l'édition de 1958; Actualités Scientifiques et Industrielles, No. 1261. MR 0417224

[Br13] Michel Brion, The coherent cohomology ring of an algebraic group, Algebr. Represent. Theory 16 (2013), no. 5, 1449-1467, DOI 10.1007/s10468-012-9364-0. MR3102962

[Br15] Michel Brion, On extensions of algebraic groups with finite quotient, Pacific J. Math. 279 (2015), no. 1-2, 135-153, DOI 10.2140/pjm.2015.279.135. MR3437773

[BSU13] Michel Brion, Preena Samuel, and V. Uma, Lectures on the structure of algebraic groups and geometric applications, CMI Lecture Series in Mathematics, vol. 1, Hindustan Book Agency, New Delhi; Chennai Mathematical Institute (CMI), Chennai, 2013. MR.3088271

[Br18] M. Brion, On the fundamental groups of commutative algebraic groups, arXiv:1805.09525, to appear at Annales Henri Lebesgue.

[CGP15] Brian Conrad, Ofer Gabber, and Gopal Prasad, Pseudo-reductive groups, 2nd ed., New Mathematical Monographs, vol. 26, Cambridge University Press, Cambridge, 2015. MR.3362817

[DG70] M. Demazure, P. Gabriel, Groupes algébriques, Masson, Paris, 1970.

[EGA] A. Grothendieck, Éléments de géométrie algébrique. I. Le langage des schémas (French), Inst. Hautes Études Sci. Publ. Math. 4 (1960), 228. MR217083

[HH19] William J. Haboush and Donghoon Hyeon, Conjugacy classes of commuting nilpotents, Trans. Amer. Math. Soc. 372 (2019), no. 6, 4293-4311, DOI 10.1090/tran/7782. $\operatorname{MR} 4009390$

[Ja03] Jens Carsten Jantzen, Representations of algebraic groups, 2nd ed., Mathematical Surveys and Monographs, vol. 107, American Mathematical Society, Providence, RI, 2003. MR:2015057

[KS05] Masaki Kashiwara and Pierre Schapira, Categories and sheaves, Grundlehren der Mathematischen Wissenschaften [Fundamental Principles of Mathematical Sciences], vol. 332, Springer-Verlag, Berlin, 2006. MR2182076

[La11] Adrian Langer, On the S-fundamental group scheme (English, with English and French summaries), Ann. Inst. Fourier (Grenoble) 61 (2011), no. 5, 2077-2119 (2012), DOI 10.5802/aif.2667. MR.2961849

[La12] Adrian Langer, On the S-fundamental group scheme. II, J. Inst. Math. Jussieu 11 (2012), no. 4, 835-854, DOI 10.1017/S1474748012000011. MR2979824

[Lu17] Giancarlo Lucchini Arteche, Extensions of algebraic groups with finite quotient and nonabelian 2-cohomology, J. Algebra 492 (2017), 102-129, DOI 10.1016/j.jalgebra.2017.08.026. MR.3709145

[Mi73] Masayoshi Miyanishi, Some remarks on algebraic homogeneous vector bundles, Number theory, algebraic geometry and commutative algebra, in honor of Yasuo Akizuki, Kinokuniya, Tokyo, 1973, pp. 71-93. MR0360588

[Mu78] Shigeru Mukai, Semi-homogeneous vector bundles on an Abelian variety, J. Math. Kyoto Univ. 18 (1978), no. 2, 239-272, DOI 10.1215/kjm/1250522574. MR498572

[Mu08] David Mumford, Abelian varieties, Tata Institute of Fundamental Research Studies in Mathematics, vol. 5, Published for the Tata Institute of Fundamental Research, Bombay; by Hindustan Book Agency, New Delhi, 2008. With appendices by C. P. Ramanujam and Yuri Manin; Corrected reprint of the second (1974) edition. MR2514037

[No82] Madhav V. Nori, The fundamental group-scheme, Proc. Indian Acad. Sci. Math. Sci. 91 (1982), no. 2, 73-122, DOI 10.1007/BF02967978. MR682517

[No83] Madhav V. Nori, The fundamental group-scheme of an abelian variety, Math. Ann. 263 (1983), no. 3, 263-266, DOI 10.1007/BF01457128. MR704291

[Oo66] F. Oort, Commutative group schemes, Lecture Notes in Mathematics, vol. 15, SpringerVerlag, Berlin-New York, 1966. MR0213365

[Pe75] Daniel Perrin, Schémas en groupes quasi-compacts sur un corps (French), Schémas en groupes quasi-compacts sur un corps et groupes henséliens, U. E. R. Math., Univ. Paris XI, Orsay, 1975, pp. 1-75. MR0409487 
[Pe76] Daniel Perrin, Approximation des schémas en groupes, quasi compacts sur un corps, Bull. Soc. Math. France 104 (1976), no. 3, 323-335. MR 432661.

[ST16] Akiyoshi Sannai and Hiromu Tanaka, A characterization of ordinary abelian varieties by the Frobenius push-forward of the structure sheaf, Math. Ann. 366 (2016), no. 3-4, 1067-1087, DOI 10.1007/s00208-015-1352-3. MR.3563232

[Sc10] Stefan Schröer, On the ring of unipotent vector bundles on elliptic curves in positive characteristics, J. Lond. Math. Soc. (2) $\mathbf{8 2}$ (2010), no. 1, 110-124, DOI 10.1112/jlms/jdq028. MR2669643

[SGA3] Schémas en groupes. I: Propriétés générales des schémas en groupes (French), Séminaire de Géométrie Algébrique du Bois Marie 1962/64 (SGA 3). Dirigé par M. Demazure et A. Grothendieck. Lecture Notes in Mathematics, Vol. 151, Springer-Verlag, Berlin-New York, 1970. MR0274458

[Se60] Jean-Pierre Serre, Groupes proalgébriques (French), Inst. Hautes Études Sci. Publ. Math. 7 (1960), 67. MR 118722

[Se97] Jean-Pierre Serre, Algebraic groups and class fields, Graduate Texts in Mathematics, vol. 117, Springer-Verlag, New York, 1988. Translated from the French. MR918564

[SP19] The Stacks Project Authors, Stacks Project, http://stacks.math.columbia.edu, 2019.

[To13] Burt Totaro, Pseudo-abelian varieties (English, with English and French summaries), Ann. Sci. Éc. Norm. Supér. (4) 46 (2013), no. 5, 693-721, DOI 10.24033/asens.2199. MR.3185350

[Wa79] William C. Waterhouse, Introduction to affine group schemes, Graduate Texts in Mathematics, vol. 66, Springer-Verlag, New York-Berlin, 1979. MR547117

[Wu86] Xiao Long Wu, On the extensions of abelian varieties by affine group schemes, Group theory, Beijing 1984, Lecture Notes in Math., vol. 1185, Springer, Berlin, 1986, pp. 361387, DOI 10.1007/BFb0076184. MR842453

Institut Fourier, Université de Grenoble, 100 Rue des Mathématiques, 38610 Gières, FRANCE

Email address: Michel.Brion@univ-grenoble-alpes.fr 